[Contribution from the Research Laboratory of the General, Elemctric COMPANY.]

\title{
THE ARRANGEMENT OF ELECTRONS IN ATOMS AND MOLECULES.
}

\author{
BY IRviNg LANGMUIR. \\ Received March 3, 1919.
}

The problem of the structure of atoms has been attacked mainly by physicists who have given little consideration to the chemical properties which must ultimately be explained by a theory of atomic structure. The vast store of knowledge of chemical properties and relationships, such as is summarized by the Periodic Table, should serve as a better foundation for a theory of atomic structure than the relatively meager experimental data along purely physical lines.

Kosse $^{1}$ and Lewis ${ }^{2}$ have had marked success in attacking the problem in this way. The present paper aims to develop and somewhat modify these theories. Lewis, rejecting the physical data as being insufficient or inconclusive, reasons from chemical facts that the electrons in atoms are normally stationary in position. These electrons arrange themselves in a series of concentric shells, the first shell containing two electrons, while all other shells tend to hold eight. The outermost shell however may hold 2,4 , or 6 , instead of 8 . The 8 electrons in a shell are supposed to be placed symmetrically at the corners of a cube or in pairs at the corners of a regular tetrahedron. When atoms combine they usually hold some of their outer electrons in common, two electrons being thus held for each chemical bond. These electrons may form parts of both atomic shells of 8 electrons. By means of these postulates Lewis is able to give an extraordinarily satisfactory explanation of the periodic arrangement of the elements and to explain in detail most of their chemical properties. $\mathrm{He}$ confines his attention, however, exclusively to the inert gases, the alkali and the alkaline earth metals, the halogens, boron, aluminium, scandium, carbon, silicon, nitrogen, phosphorus, arsenic, antimony, bismuth, oxygen, sulfur, selenium, and tellurium, a total of 35 out of the 88 known elements. The theory in its present form does not apply at all satisfactorily to any of the other elements.

Kossel's theory has many points of similarity. He conceives of the electrons as located in a plane in concentric rings, rotating in orbits about the nucleus. Certain arrangements, corresponding to those of the inert gases, are supposed to be of unusual stability and all the other atoms, in forming compounds tend to give up or take up electrons so that their electrons may become arranged like those of the inert gases. Kossel considers only the elements up to cerium, a total of 57 . His theory does

i W. Kossell, Ann Physik, 49, 229 (I9I6).

"G. N. Lewis, This Journal, 38, 762 (ig16). 
not satisfactorily account for the properties of the elements from vanadium to zinc or from columbium to silver and is only partially satisfactory for any of the elements above vanadium. In other words, its main success is limited to the first 23 elements. The theory does not lend itself nearly as well as that of Lewis to the detailed explanation of the properties of elements and their compounds. A rather thorough review and discussion of these and other recent theories of atomic structure has been published by S. Dushman. ${ }^{1}$

There is much chemical evidence, especially in the field of stereochemistry, that the primary valence forces between atoms act in directions nearly fixed with respect to each other. This can only be satisfactorily accounted for by electrons arranged in three dimensions.

Kossel attempts to explain the tetrahedral arrangement of the carbon valences by arguing that 4 spheres drawn in by strong forces towards a central atom must arrange themselves as a tetrahedron and that if the forces are great enough they will not be able to shift their positions. It is evident that even this structure would not have the requisite symmetry for the carbon atom, when the plane of the electron orbit is taken into account. But there is, moreover, conclusive evidence, even when carbon atoms are surrounded by less than 4 other atoms, that the forces act in definite directions. For example, if wood is carbonized under certain conditions a charcoal is obtained having about the same volume as the wood. This is notably true when such a substance as finely divided tungsten trioxide is reduced in very dry hydrogen. The volumes occupied are in some cases 20 or 25 times as great as that of the corresponding solid in crystalline form. The whole behavior of such bodies, especially in regard to their sintering at higher temperatures, indicates that the atoms are arranged in branching chains in which most atoms are surrounded by only two or three others. Since the bodies are definitely solid it must follow that the atoms are not able to shift their relative positions except when acted on by strong external forces. Such structures are inconceivable if atoms contain only electrons revolving in orbits about the nuclei.

Further evidence for the stationary electrons has been obtained by Hull who finds that the intensities of the lines in the X-ray spectra of crystals are best accounted for on the theory that the electrons occupy definite positions in the crystal lattice.

In attempting to determine the arrangement of electrons in atoms we must be guided by the numbers of electrons which make up the atoms of the inert gases; in other words by the atomic numbers of these elements, namely, helium 2, neon 10, argon 18, krypton 36 , xenon 54 , and niton 86.

1 Gen. Elec. Rev., 20, 186, 397 (1917). 
Rydberg ${ }^{1}$ has pointed out that these numbers are obtained from the series

$$
N=2\left(\mathrm{I}+2^{2}+2^{2}+3^{2}+3^{2}+4^{2}+\right) .
$$

The factor two suggests a fundamental two-fold symmetry for all stable atoms. By a consideration of this equation and principles of symmetry and by constant checking against the Periodic Table and the specific properties of elements I have been led to the postulates given below. Some of these may seem in themselves to be very improbable and will undoubtedly need to be modified as more facts are acquired. But it is felt that all contain a fundamental basis of truth and that, although future modifications may make them take rather different forms, their application in predicting properties of elements will not be greatly altered.

The first postulate is concerned particularly with the structure of the stable atoms of the inert gases.

Postulate I.-The electrons in the atoms of the inert gases are arranged about the nucleus in pairs symmetrically placed with respect to a plane passing through the nucleus which we may call the equatorial plane. The atoms are symmetrical with respect to a polar axis perpendicular to the plane and passing through the nucleus. They have also 4 secondary planes of symmetry passing through the polar axis and making $45^{\circ}$ angles with each other. The symmetry thus corresponds to that of a tetragonal crystal. Since the electrons must occur in pairs symmetrical to the equational plane there are no electrons in this plane.

Postulate 2.-The electrons in the atoms are distributed through a series of concentric spherical ${ }^{2}$ shells. All the shells in a given atom are of equal thickness. If the mean of the inner and outer radii be considered to be the effective radius of the shell then the radii of the different shells stand in the ratio $1: 2: 3: 4$, and the effective surfaces of the shells are in the ratio $1: 2^{2}: 3^{2}: 4^{2}$.

Postulate 3.-Each spherical shell is divided into a number of cellular spaces. The thickness of these cells measured in a radial direction is equal to the thickness of the shell and is therefore the same (Postulate 2) for all the cells in the atom. In any given atom the cells occupy equal areas in their respective shells. All the cells in an atom have therefore equal volumes. The first postulate, regarding symmetry, applies also to the location of the cells. The first shell therefore contains two cells obtained by dividing the shell into two equal parts

1 Phil. Mag., 28, 144 (Ig14).

2 In accordance with Postulate $r$, it is probable that the surfaces of the shells are ellipoids of revolution rather than spheres. In the present argument this distinction is immaterial. 
by the equatorial plane. The second shell having 4 times the surface (Postulate 2) contains 8 cells. The third shell thus contains 18 while the fourth contains 32 cells. Or if we consider only one hemisphere the numbers in the successive shells are I, 4, 9 and 16.

Postulate 4.-Each of the two innermost cells can contain only one electron ${ }^{1}$ but each of the other cells is capable of holding two. There can be no electrons in the outside shell until all the inner shells contain their maximum numbers of electrons. In the outside shell two electrons can occupy a single cell only when all other cells contain at least one electron. We may assume that two electrons occupying the same cell are at different distances from the nucleus. Each shell, containing its full quota of electrons, thus consists of two "layers." We will find it convenient to refer to these layers of electrons by the symbols I, II $a, \operatorname{II} b, \operatorname{III} a, \operatorname{III} b$, and IV $a$ where the Roman numerals denote the shell containing the layer. Helium, neon, argon, krypton, or xenon, contains, respectively, the first $1,2,3$, 4 , or 5 of these layers, while niton contains all six.

The two-fold symmetry assumed in Postulate $I$ is derived from the factor 2 which occurs in Rydberg's equation. The 4 -fold symmetry is derived from the remarkable numerical relation brought out in the following table:

\begin{tabular}{|c|c|c|c|c|}
\hline \multirow{2}{*}{ Shell. } & \multirow[b]{2}{*}{ Radius. } & \multirow[b]{2}{*}{$n}$. & \multicolumn{2}{|c|}{ No. of cellis } \\
\hline & & & in axis. & in zones \\
\hline I... & . $\mathrm{I}$ & I & I & 0 \\
\hline II. . & 2 & 4 & o & 4 \\
\hline III. & 3 & 9 & I & 8 \\
\hline IV.. & 4 & I 6 & 0 & 16 \\
\hline
\end{tabular}

Here $n$ represents the number of cells in one of the hemispheres of the shell. If this number is odd one of the cells must lie along the polar axis; all other cells must be distributed in zones about this axis.

We see from this table that the number of cells which must be arranged in zones is always a multiple of 4 . We can therefore assume tetragonal symmetry for the atoms of the inert gases.

Postulates 2 and 3 offer perhaps the simplest possible explanation of the occurrence of the terms $1,2^{2}, 3^{2}$ and $4^{2}$, in Rydberg's relation. There are some reasons for believing that the shells close to the nucleus would lie closer together. These reasons are based mainly on the assumption that Coulomb's inverse square law holds even at short distances, and for this assumption there is little experimental evidence, except in the case of forces between two positive nuclei (Rutherford's scattering

1 If, as Rydberg believes, there are two undiscovered elements of atomic weights less than that of hydrogen, then this exception in the case of the innermost cells may be avoided. 
experiments). It is probable that the law of force is quite different for electrons bound in an atom and for positive or negative particles passing through the atom.

The assumption of the existence of cells independent of the electrons in them, seems to be needed to account for the properties of elements above the rare earths. It is however closely related to Bohr's assumption of the existence of stationary states. The passage of an electron from one cell to another probably causes the emission of a spectrum line. It should be noted that the numbers $\mathrm{I}, 2^{2}, 3^{2}, 4^{2}$, etc., also occur in Bohr's theory in the determining of the location of the stationary orbits. It is probable that a common explanation will be found for both theories.

Postulate 4 seems necessary to take into account that the terms $2^{2}$, $3^{2}$, etc., in Rydberg's series occur twice. It seems to denote a remarkable tendency like that suggested by Postulate I for the electrons to form pairs.

From the steady progression in the properties of the different inert gases, however, we must conclude that the two electrons in a single cell do not exert very strong forces on each other.

The first 4 postulates give us a definite conception of the arrangement of the electrons in the atoms of the inert gases. Helium consists of two electrons symmetrically placed with respect to the nucleus. This same pair exists (as Shell I) in the atoms of all the other inert gases and determines the position of the polar axis. Neon contains a second shell (II a) containing 8 electrons arranged at the corners of two squares placed symmetrically with respect to the equatorial plane and parallel to it. These positions probably correspond fairly closely to the corners of a cube, but the effect of the two electrons in the first shell should be to shorten the cube in the direction of the polar axis. Argon is just like neon, except that there is a second layer of 8 electrons (IIb) in the second shell. The two inner shells of krypton are like the two shells of argon, but in addition it has a third shell containing 18 electrons. Two of these electrons are located at the ends of the polar axis while the other 16 are placed symmetrically to the axis and to the equatorial plane and to the electrons in the inner shells. In all probability 4 of the 8 electrons in each hemisphere are located in the same plane as those in the second shell, while the other 4 are in planes making a $45^{\circ}$ angle with these.

Xenon is like krypton except for the addition of another layer (IIIb) of electrons in the third shell.

Niton has in addition to the 3 shells of xenon a fourth shell containing 32 electrons, 16 for each hemisphere. We have no data by which to determine the exact arrangement of these, but it is obvious that the $I 6$ can be arranged with a high degree of symmetry with respect to the underlying layer of 8 electrons (in each hemisphere).

The following postulates deal with the forces and tendencies which 
govern the arrangement of electrons in the outside layer of atoms other than those of the inert gases:

Postulate 5.-It is assumed that electrons contained in the same cell are nearly without effect on each other. But the electrons in the outside layer tend to line themselves up (in a radial direction) with those of the underlying shell because of a magnetic field probably always to be associated with electrons bound in atoms. (Parson's magneton theory.) This attraction may be more or less counteracted by the electrostatic repulsion between the outside electrons and those in the underlying shell. The electrons in the outside layer also repel each other and thus tend to distribute themselves among the available cells so as to be as far apart as possible. The actual positions of equilibrium depend on a balance between these 3 sets of forces together with the attractive force exerted by the nucleus.

Postulate 6.-When the number of electrons in the outside layer is small, the magnetic attraction exerted by the electrons of the inner shells tends to predominate over the electrostatic repulsion, but when the atomic number and the number of electrons in the outside layer increase, the electrostatic forces gradually become the controlling factor. As a result, when there are few electrons in the outer layer these arrange themselves in the cells over those of the underlying shell, but where the outside layer begins to approach its full quota of electrons the cells over the underlying electrons tend to remain empty.

Postulate 7.-The properties of the atoms are determined by the number and arrangement of electrons in the outside layer and the ease with which they are able to revert to more stable forms by giving up or taking up electrons, or by sharing their outside electrons with atoms with which they combine. The tendencies to revert to the forms represented by the atoms of the inert gases are the strongest, but there are a few other forms of high symmetry such as those corresponding to certain possible forms of nickel, palladium, erbium and platinum atoms towards which atoms have a weaker tendency to revert (by giving up electrons only).

We may now apply these 7 postulates to derive the properties of the chemical elements. We will first go through the list of elements dealing only with broad features and will later consider the properties of certain elements in more detail. At present we will confine our attention to the properties of the elements in atomic condition-we shall discuss only their tendencies to take up or give up electrons. The properties of the elements in solid or liquid form or in their compounds involve forces acting between different atoms and therefore can be best considered after 
we have discussed the formation of molecules. The properties of the atoms up to argon fit in well with even the older arrangements of the periodic table. In fact, the present theory and Lewis' theory resemble each other very closely as far as their application to these first 18 elements is concerned.

TABLE 1 .

Classification of the Elements According to the Arrangement of Their Electrons.

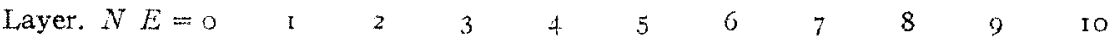
I

II 2 He $\quad \mathrm{Li} \quad \mathrm{Be} \quad \mathrm{B}$

II $b$ ro $\mathrm{Ne} \quad \mathrm{Na} \quad \mathrm{Mg} \quad \mathrm{Al}$

C N O F $\mathrm{F}$ Ne

III I $^{\mathrm{A}} \mathrm{K}$ Ca Se

Si $\quad \mathrm{P} \quad \mathrm{S} \quad \mathrm{Cl} \quad \mathrm{A}$

\begin{tabular}{|c|c|c|c|c|c|c|c|c|c|c|c|c|}
\hline$\Pi \pi$ & I 8 & $A$ & $\mathrm{~K}$ & $\mathrm{Ca}$ & Se & $\mathrm{Ti}$ & V & $\mathrm{Cr}$ & $\mathrm{Mn}$ & $\mathrm{Fe}$ & Co & $\mathrm{Ni}$ \\
\hline & & & 11 & 12 & 13 & 14 & 15 & 16 & 17 & 18 & & \\
\hline $\operatorname{III}_{e}$ & 28 & $\mathrm{Ni} \beta$ & $\mathrm{Cu}$ & $\mathrm{Zn}$ & $\mathrm{Ga}$ & $\mathrm{Ge}$ & As & $\mathrm{Se}$ & $\mathrm{Br}$ & $\mathrm{Kr}$ & & \\
\hline III $b$ & 36 & $\mathrm{Kr}$ & $\mathrm{Rb}$ & $\mathrm{Sr}$ & $Y$ & $\mathrm{Zr}$ & $\mathrm{Cb}$ & Mo & 43 & $\mathrm{R} u$ & $\mathrm{Rh}$ & $\mathrm{Pd}$ \\
\hline & & & 11 & 12 & 13 & 14 & 15 & 16 & 17 & 18 & & \\
\hline III $b$ & 46 & $\mathrm{Pd} \beta$ & Ag & $\mathrm{Cd}$ & In & $\mathrm{Sn}$ & $\mathrm{Sb}$ & $\mathrm{Te}$ & I & $\mathrm{Xe}$ & & \\
\hline IVa & 54 & $\mathrm{Xe}$ & $C s$ & $\mathrm{Ba}$ & Ia & $\mathrm{Ce}$ & $\operatorname{Pr}$ & $\mathrm{Nd}$ & $6 \mathrm{I}$ & $\mathrm{Sa}$ & $\mathrm{Eu}$ & Gd \\
\hline & & & 11 & 12 & 13 & 14 & 15 & 16 & 17 & 18 & & \\
\hline IV $a$ & & & $\mathrm{~Tb}$ & Ho & Dy & 玨 & $\mathrm{Tm}$ & $1 \mathrm{~m}_{2}$ & $\mathrm{Yb}$ & Lu & & \\
\hline & & 14 & 15 & 16 & 17 & 18 & 19 & 20 & 21 & 22 & 23 & 24 \\
\hline IV $a$ & 68 & $\operatorname{Lir} \beta$ & $\operatorname{Tm} \beta$ & $\mathrm{T}^{4} \mathrm{~m}_{2} \mathrm{\theta}$ & $\mathrm{Yb} \beta$ & $\operatorname{Lu} \beta$ & $\mathrm{Ta}$ & $W$ & 75 & Os & Ir & $P t$ \\
\hline & & & 25 & 26 & 27 & 28 & 29 & 30 & 31 & 32 & & \\
\hline IVa & 78 & $\mathrm{Pt} \beta$ & $\mathrm{Au}$ & Hg & $\mathrm{Tl}$ & $I b$ & $\mathrm{Bi}$ & $\mathrm{RaF}$ & 85 & $\mathrm{Nt}$ & & \\
\hline IV $b$ & 86 & $\mathrm{Nt}$ & 87 & $\mathrm{Ra}$ & Ac & $\mathrm{Th}$ & $\mathrm{Ux}_{2}$ & U & & & & \\
\hline
\end{tabular}

Table I to which it will be convenient to refer frequently during the following discussion, contains a list of all the elements, arranged in order of their atomic numbers. The table is designed to show the way in which the electrons are arranged in the different shells. The numbers forming the first horizontal line denote the number of electrons in the outside layer of the atom. The first vertical column gives the index number of this outside layer. Thus boron has 3 electrons in the II a layer, chromium has 6 in the III a layer.

Hydrogen ( $N=I$ ) has a single electron. It is therefore (Postulates I and 7) unsaturated and tends to take up an electron in order to assume the symmetrical form characteristic of helium. The valence of hydrogen is therefore unity.

With helium $(N=2)$ the first shell is completed. Beyond this point any additional electrons must go into the first layer of the second shell 
(IIa). There are 8 cells in this layer (Postulate 3 ) so that 8 electrons can be added before the atoms again acquire the stability of an inert gas. In lithium $(N=3)$ the single electron in the second shell is easily detached so that the atom reverts to the stable form that corresponds to helium, thus forming a univalent cation. In Fig. I the positive and negative valences of the elements are plotted against their atomic numbers. ${ }^{1}$ It is seen that up to $N=$ I7 the maximum positive valency increases regularly up to the halogens (with the exception of oxygen and fluorine). This maximum valency is determined by the number of electrons which are given up when the atom reverts to that of the next lower inert gas.

In beryllium and boron the properties are determined largely by the ability of the atom to revert to the form corresponding to helium. The actual arrangement of the electrons in the atoms of these elements is thus of little significance. In carbon the 4 electrons in the second shell tend to arrange themselves (Postulate 5) at the corners of a tetrahedron for in this way they can get as far apart as possible. With nitrogen no symmetrical arrangement of the 5 electrons is possible. We shall see that this leads nitrogen to form a series of very unusual compounds. Whereas the properties of the elements from lithium up to carbon vary in a rather regular progression, the properties of carbon and nitrogen form a very sharp discontinuity. The constant valence of carbon-the variable valence of nitrogen; the high melting point of carbonthe low melting point of nitrogen; the very great inertness and stability of most carbon compounds-the very great activity and often explosive properties of nitrogen compoundsall these illustrate this fundamental break in properties. We shall see that a somewhat

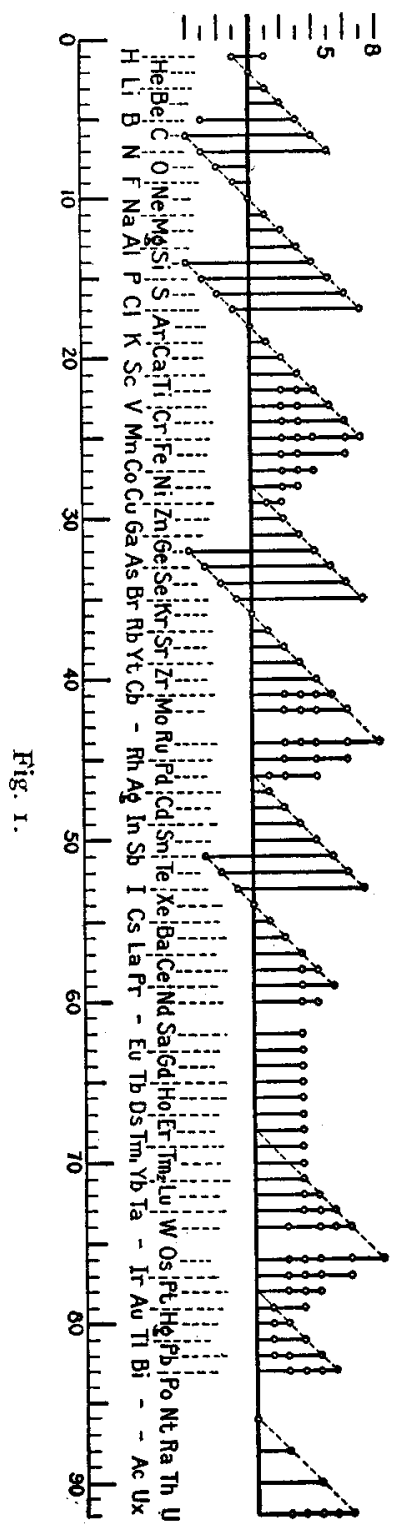
similar break occurs in each case where the atom becomes equally unsym- 1 This figure is taken with some modifications from I. W. D. Hackh. THIS JoURNAL, 40, 1024 (I9I8). 
metrical, namely in the fifth element of each succeeding shell, thus the breaks occur at nitrogen (II $a$ ) phosphorus (II $b$ ), vanadium (III $a$ ), columbium (IIIb), praseodymium (IVa).

\section{First Long Period.}

Beyond argon we soon come into a region where most periodic relations begin to fail. It will therefore be well to examine the present theory rather critically.

With potassium we begin to form a new shell-the third. There are now cells enough to hold 9 electrons in each hemisphere. (Postulate 3.) The first few electrons arrange themselves in much the same way as in the first two periods. Thus potassium, calcium, scandium have properties closely related to those of sodium, magnesium and aluminum. In the first two periods the properties of the atoms just beyond carbon and silicon were electronegative in character because their atoms tended to assume the stable forms corresponding to neon and argon by taking up electrons. But in the third period the conditions are quite different. Thus in the atoms of the eighth element of this period, iron, only 4 out of the 9 outside cells in each hemisphere contain electrons. This atom therefore does not have the stability of those of the inert gases and there is thus little tendency for the elements of lower atomic number to take up electrons. The elements titanium, vanadium, chromium, manganese, have therefore predominantly electropositive character. In Table I the differences between the properties of these elements and those of the corresponding elements in the earlier periods is indicated by the heavy lines enclosing these elements. These lines also express the mutual resemblance between the elements.

We can go much further in predicting the properties. The maximum valence of the elements is determined by the ability of their atoms to revert to argon, thus vanadium has a valency of 5 , chromium 6 , and manganese 7 . It will be shown that in the formation of acid radicals with high valences the electrons, although they are given up to oxygen atoms, yet remain within the field of force of the original atom. In other words, the acid-forming atoms share their electrons with oxygen atoms but do not lose them completely. In compounds in which these high valences occur the properties are like those of the corresponding elements of the previous period. Thus vanadates resemble phosphates, chromates resemble sulfates and permanganates are like perchlorates. But when these elements have other valences their properties do not show such relationships.

The electropositive character of potassium, calcium and scandium is determined by the ease with which they revert to argon. Elements like vanadium, chromium and manganese, however, cannot form positive ions by reverting to argon for they would have to give up completely 5 , 
6 and 7 electrons, respectively. The large electrostatic forces involved prevent the formation of ions with such large charges. The electropositive character of these elements must manifest itself therefore by the formation of ions with fewer charges. The tendency to give up electrons is dependent on the presence of electronegative elements capable of taking up the electrons. ${ }^{1}$ In general, we may suppose that the atoms in a metal are held to each other by very strong forces as indicated for example by the high heats of evaporation. If the metal goes into solution a large amount of energy must be expended in separating these atoms and in removing some of their electrons. This energy is supplied by the energy of combination of the electronegative elements with the electrons separated from the atoms when they go into solution in the form of ions. Now if a metal forms divalent ions, more energy can be supplied than if it forms univalent ions. On the other hand it will be more difficult to separate two electrons than one electron from an atom. Thus we may suppose in a given case that univalent ions will not form because the energy supplied by the combination of a single electron with the electronegative atom is not sufficient to separate the metal atom from the others. On the other hand quadrivalent ions may not form because the energy necessary to separate 4 electrons from the atom may be greater than that which can be supplied by the combination of the 4 electrons with the electronegative element. $^{2}$ It may happen however that both divalent and tervalent ions can form with about equal ease.

1 The form sometimes given to Nernst's theory by which the tendency to form ions is due to a solution pressure cannot correspond to the true mechanism of ionization. The real tendency of such a substance as potassium to give off electrons is measured by the Richardson work function as determined from the electron emission in high vacuum. The tendency for single atoms of potassium to give off electrons is measured by the ionizing potential. In both cases ąn energy must be expended to separate electrons from potassium which corresponds to a difference of potential of a couple of volts. I have previously discussed at some length the general theory underlying this statement (Langmuir, Trans. Am. Electrochem. Soc., 29, 125 (1916)).

2 It should be kept in mind that the work which must be done to separate electrons from an atom increases rapidly with the number removed. Thus if $\mathrm{I}$ represents the work done to remove one electron, the work done to remove the second is 2, the third 3 and the fourth 4 . To remove 4 electrons the total work is thus Io times that needed to remove one. The energy supplied by the formation of the anions increases however in direct proportion to the number of electrons removed. To take a concrete example, let us assume that in terms of the energy units chosen above the formation of each anion supplies 2.7 units, while the separation of the metal atoms from each other requires the expenditure of 2 units per atom. Then the free energy supply and consumption for the differently charged cations are

$\begin{array}{lcccccc}\text { Number of Electrons } & 0 & 1 & 2 & 3 & 4 & 5 \\ \text { Energy supplied ............. } & 0 & 2.7 & 5.4 & 8.1 & 10.8 & 13.5 \\ \text { Energy consumed............ } & 2.0 & 3.0 & 5.0 & 8.0 & 12.0 & 17.0\end{array}$

In this case therefore there is sufficient energy to form the divalent or trivalent ion but not sufficient to form the univalent, quadrivalent or quinquivalent ion. 
As a matter of fact, if we examine Fig. I we see that the elements vanadium, chromium, manganese, iron, all form divalent and tervalent cations but form no univalent or quadrivalent ions in solution. The fact that they all form ions of the same valence with so nearly the same ease shows that the stability of these electrons in these atoms is very nearly the sane. But this is just what our theory would lead us to expect.

For the elements under consideration only 5 to 8 out of the is cells in the third shell are filled with electrons. Furthermore, the tendency of the electrons to line up (Postulate 6) with the underlying electrons of the second shell is gradually being weakened by the mutual electrostatic repulsion. Therefore, the tendencies of these elements to give up electrons do not differ greatly. The lack of definite forces to determine the distribution of the electrons among the cells, renders these elements (according to a theory of Lewis) ${ }^{1}$ capable of absorbing light in the visible spectrum. We thus find that they all form colored salts. To quote from Lewis: "The difficuity **** lies in the fact that the kernel of these atoms is not uniquely and permanently defined. It seems probable that in these elements there is a possibility of the transfer of electrons either from one part of the kernel to another, or between the kernel and the outer shell, or possibly between two separate outer shells of the same atom and that electrons which are suspended midway between two such stages are responsible for this absorption of light in these cases." According to the present theory the absorption is caused by the transfer of electrons between different parts of the same outside shell or is possibly due to the ease with which an electron is gained or lost by the outside shell. There is no necessity for, and in fact every probability against, the kernel ( $i$. e., the inner shells) being concerned in this process.

In the atoms of iron there are 8 electrons in the third shell, or 4 in each hemisphere. According to Postulate 6 the magnetic forces will make these electrons take positions as close as possible to those of the underlying shell. We may therefore picture the structure of the iron atom as follows: Close to the nucleus are two electrons. The 24 remaining electrons arrange themselves in 3 layers at the corners of 3 concentric cubes (slightly flattened) whose diagonals coincide. Although this atom possesses about as high a degree of symmetry as that of argon it differs radically from the latter in that the outer shell is not saturated, only 4 of the 9 cells in each hemisphere being occupied by electrons (Postulate 3 . Furthermore, the number of electrons in the outside shell is beginning to be so great that the electrostatic repulsion (Postulate 6) tends to decrease the stability of this arrangement. These are the funda* mental reasons that the elements of smaller atomic number like chromium

Sce also Bichowsky, JuIs Journat, 40, 500 (1918). 
and manganese do not exhibit the electronegative properties of sulfur and chlorine.

In cobalt and nickel whose atomic numbers are, respectively, one and two units larger than that of iron, the extra electrons can no longer be arranged over those in the underlying shell. Let us consider the way that the electrons in nickel arrange themselves. In the outside shell there are 5 electrons in each hemisphere and these tend to arrange themselves over the underlying 4 electrons (II b). The only position of reasonable symmetry which the extra electron can take is directly over the center of the square formed by the 4 electrons of the second shell. In other words, the electron goes into the polar axis of the atom. In cobalt there is an electron at one end of the polar axis but not at the other.

The present theory thus explains in a perfectly satisfactory way the anomalous position of iron, cobalt and nickel in the periodic table. We shall see that it also accounts for their unusual magnetic properties.

The ordinary chemical properties of these 3 elements resemble those of chromium and manganese, except that they have lost most. of the acidforming properties because they are so far removed from argon that they cannot revert to it. Thus these 3 elements never show valences of 8,9 , and io and do not form acids corresponding to chromates and permanganates. They form predominantly salts in which they exist as divalent or tervalent cations and for the same reasons as those discussed in connection with vanadium, chromium and manganese. The colors of their salts are even more marked than those of the chromium and manganese cations which indicates that their electrons are even more loosely bound. With nickel the number of electrons is so great that the electrostatic forces seriously oppose the magnetic forces (Postulate 6). The introduction of the electrons in the polar axis also tends to force the other electrons away from their positions over the underlying electrons and thus still further weakens the magnetic forces. The effect is thus to bring about a rearrangement of the electrons so that the square containing the 4 electrons in each hemisphere, tends to revolve $45^{\circ}$ about the polar axis. This arrangement, which we may call the $\beta$-form, has a higher degree of symmetry than the $\alpha$-form previously considered, in that all the electrons in the $\beta$-form are as far from the underlying ones as possible, while in the $\alpha$-form one electron was far from, and the other 4 were close to, the inner ones.

According to Postulate 3 we should look upon the transformation from the $\alpha$-to the $\beta$-form as involving the passage of electrons between different cells in the outside layer. It is perhaps best to imagine that it is the empty cells which arrange themselves over the underlying electrons in the $\beta$-form.

The $\beta$-form of the nickel atom has a symmetry which exceeds that of 
any other atom between argon and krypton, with the possible exception of iron. Thus the atoms of the elements above nickel in giving up electrons tend to revert to either nickel or iron. But by the time we get to elements above nickel the large nuclear charge causes the electrostatic forces to predominate over the magnetic so that the tendency to revert to iron is eliminated.

As a matter of fact, by referring to Fig. I, we find that copper (cuprous) has a valency of $I$, zinc has 2 , gallium 3 , etc., right up to selenium, 6. These elements therefore all tend to give up electrons in such a way that their atoms revert back to the symmetrical $\beta$-form of the nickel atom. It must be remembered that after these atoms have given up their extra electrons their outside layers contain the same number of electrons as the nickel atoms but they differ from the latter in that the charge on the nucleus is greater and therefore there is a much greater tendency for the $\beta$-form to be the more stable form.

The tendency of the elements above nickel to revert to the $\beta$-form of nickel is expressed in Table I by placing $\mathrm{Ni} \beta$ in the same vertical column as the inert gases. The horizontal lines enclosing these elements indicate the distinction between the structure of $\mathrm{Ni} \beta$ and atoms of the inert gases.

In the case of copper we find that the ability to form an ion having an $\alpha$-form is not wholly lost. Thus copper forms divalent ions. The cupric ions form a continuation of the family of similar elements which extended from vanadium to nickel, but the cuprous salts form a radical departure from this series. The electrons are given up in the formation of cupric ions not because there is any inherent stability in the cobalt atom to which it reverts but for the same reason that chromium, manganese, iron, nickel and cobalt form divalent ions. It is interesting to note that the cupric salts are highly colored and in many ways resemble nickel salts. The cuprous salts on the other hand resemble those of no element thus far considered. It is true the atoms have the same valence as those of the alkali metals and that the ions are colorless in both cases showing that all electrons are firmly held. But the solubilities of the salts are very radically different. Now this is just the kind of difference we should expect from the difference between the structure of $\mathrm{Ni} \beta$ and the atoms of the inert gases. The atoms of these gases are characterized by weak secondary valence forces (low boiling points, etc.) whereas atoms having only about half of the cells in the outside shell filled have strong residual fields of force. The properties of the alkali metals are therefore determined almost wholly by the electrostatic charges on their ions, but with ions like that of univalent copper there is in addition the residual field of force due to the large number of unsaturated electrons. The 
cuprous ion thus tends to form insoluble solid salts and many addition products.

With zinc we have completely broken away from the tendency to variable valence. The salts are now all colorless. The tendency to form molecular compounds (secondary valence) still distinguished this element from the more purely electropositive metal like calcium.

Germanium is interesting since it begins to acquire an electronegative character because of its ability to assume the form of krypton by taking up 4 electrons. It thus resembles carbon and silicon in forming a volatile hydride whereas such a tendency is absent in case of titanium.

In arsenic, selenium, and bromine because of the proximity of krypton, the electronegative character predominates and these elements thus closely resemble phosphorus, sulfur, and chlorine. But their tendency to form insoluble secondary valence products distinguishes them from these other elements.

\section{Magnetic Properties. ${ }^{1}$}

Before proceeding with the discussion of the elements beyond krypton let us consider the magnetic properties of the elements of the first long period. We have seen that the structures of the atoms of iron, cobalt and nickel differ from those of all the elements so far considered in that there are 24 electrons arranged at the corners of 3 concentric cubes. Furthermore, our theory leads us to believe that they are held in these positions by magnetic forces. It is significant also that Parson ${ }^{2}$ was led to assume that the magnetic properties of iron were conditioned by the existence of 4 concentric shells of 8 electrons each and that these electrons exerted magnetic forces on each other. $\mathrm{Hull}^{3}$ in a study of the crystal structure of iron by the X-ray method found that the atoms of iron are arranged according to a centered cubic lattice, that is, each atom is surrounded by 8 others in directions corresponding to the diagonals of a cube. Hull also found that the intensities of the lines in the X-ray spectrum were best accounted for on the assumption "that 8 of the 26 electrons in each atom are arranged along the cube diagonals at a distance from the center equal to one-fourth the distance to the nearest atom. $* * * *$ If all the electrons are displaced from the center of the atom along the cube diagonals in four groups of $2,8,8,8$ at distances $1 / 32,1 / 16,1 / 8$ and $^{1} / 4$, respectively, of the distance to the nearest atom, all the observed facts are accounted for within the limit of experimental error." The structure thus proposed by Hull is identical with that to which we are led by our present theory,

1 Most of the data used in the following pages have been taken from the excellent review of Theories of Magnetism by S. Dushman (Gen. Elec. Rev., May, August, September, October and December, I9I6).

2 Smithsonian Inst. Pub., Miscel. Collections, 65, No. I I (I9I5).

Phys. Rev., 9, 84 (I9I7). 
except that we should expect the radii of the electron shells to be more uniform than the values given by Hull. However this is a point of smaller significance. It is possible that the thermal agitation of the outside electrons which are not under as strong constraints as the others may somewhat modify Hull's results. It should be noted that $\mathrm{Hull}^{1}$ has found that nickel atoms arrange themselves in a crystal in a similar way to those of iron. He has not, however, determined the probable positions of the electrons.

The ferromagnetic properties of iron, cobalt, and nickel undoubtedly depend not only on the arrangement of the electrons in the atom but also on the arrangement of the atoms with respect to each other. Hull's results indicate that there are 6 electrons in a line between the centers (nuclei) of each pair of adjacent iron atoms. The two which correspond to the outside layer of electrons in the atoms are held by weak constraints, but the inner ones are probably held at least as firmly as those in argon. The fact that the ferromagnetic properties of the metals disappear when these are heated above certain critical temperatures even without change in crystalline form indicates that the outside electrons are subject to thermal agitation which destroys the regular structure necessary for the development of ferromagnetism.

It is suggestive that the next most strongly ferromagnetic substances, besides those considered, are the Heusler alloys which consist of manganese and copper together with smaller amounts of aluminum, arsenic, etc. Copper having too many electrons to give a ferromagnetic metal may supply enough electrons to the manganese atoms to make them take a structure like that of iron. Other elements than copper, such as phosphorus or nitrogen, also give ferromagnetic alloys with manganese. Similarly chromium or vanadium, although to a lesser degree, may form magnetic alloys when combined with other elements. In all these cases it is probable not only that the outer shell of the manganese, chromium or vanadium atom takes up electrons to revert to iron, but that the atoms arrange themselves in a crystal structure which helps to bring out their magnetic properties (perhaps always the centered cubic lattice).

When iron, cobalt and nickel have been heated above their critical temperatures $\left(75^{\circ}\right.$ for iron, $1075^{\circ}$ for cobalt, and $340^{\circ}$ for nickel) they lose their ferromagnetic properties and become paramagnetic and thus resemble the metals of lower atomic number. The magnetic susceptibility of the elements of the first long period varies in a remarkable manner as the atomic number of the elements increases. ${ }^{2}$ Argon is very strongly diamagnetic, potassium is already slightly paramagnetic, and the succeed-

iPhys. Rer., I0, 69: (1917).

"A curve giving the susceptibility of all the elements as a function of the atomic number has been published by Harkins \& Hall, ThIs Journal, 38, 169 (Ig16). 
ing elements calcium, titanium, vanadium, chromium and manganese show a very rapid but steady rise in paramagnetism until we come to the strongly ferromagnetic metals iron, cobalt, nickel. Beyond nickel there is a sharp discontinuity for the next element, copper, is slightly diamagnetic. The rest of the elements up to bromine have about the same susceptibility as copper, that is, all are slightly diamagnetic.

The sharp break in the curve between nickel and copper is just what our theory would lead us to expect and it affords striking indication of the correctness of the viewpoint. Still more striking confirmation is to be had in the fact that cupric salts are rather strongly paramagnetic, while cuprous salts are diamagnetic. We have already seen that in the cuprous ion the atom reverts to the $\beta$-form of nickel, while in the cupric salts it has properties which correspond closely with those of the divalent ions of iron, cobalt and nickel. In other words, in metallic copper and in cuprous salts the electrostatic forces predominate, as we have already seen, in determining the positions of the electrons while in cupric salts the magnetic forces still play an important part.

It may be asked why argon which contains electrons arranged at the corners of cubes, does not resemble iron in its magnetic properties. According to Langevin's theory of diamagnetism and paramagnetism, the presence of electronic orbits in an atom does not in general cause paramagnetism. If the orbits are so arranged in the atom that they have a resultant magnetic moment equal to zero, then the effect of an increase in the external field is to increase the diameters of some of the orbits and decrease others in such a way that diamagnetism results. It is only when the external field is able to change the direction of the axis of rotation that paramagnetism can occur. In the atoms of the inert gases the electrons (or magnetons since we assume they have a magnetic field of their own) being under very large constraints, arrange themselves so that the magnetic field of the atoms is nearly wholly internal, in other words, so that the magnetic moment is zero. Such atoms are diamagnetic. It is only where the electrons are under much weaker constraints, but yet are held by magnetic rather than by electrostatic forces that we should expect paramagnetism.

\section{Second Long Period.}

Beyond krypton the second layer in the third shell begins to be filled. The first of the I8 electrons needed to complete this layer (Postulates 3 and 4 ) arrange themselves with respect to the 8 electrons in the second shell (Postulate 5), just as in the first long period, so that ruthenium has a structure analogous to iron. The 18 electrons in the first layer of the third shell, however, make the symmetry much less perfect than it was in the case of iron. The properties of these elements are more complicated than those of the first long period and there is a greater tendency to form 
insoluble salts and secondary valence compounds. The elements up to ruthenium are slightly diamagnetic or slightly paramagnetic, but from ruthenium to palladium there is a large increase in susceptibility. This reaches a sharp maximum with palladium and then drops suddenly to a negative value for silver. There is thus the same marked discontinuity as was observed between nickel and copper. But the susceptibility of palladium is only about equal to that of manganese and is thus of quite a different order of magnitude from that of iron, cobalt, or nickel.

According to our theory there can be no doubt but that the extra two electrons in palladium arrange themselves in the polar axis. In the elements beyond this point the electrons around the polar axis seek positions as far as possible from the electrons in the second shell, so that the atoms tend to revert to a $\beta$-form of the palladium atom. Thus silver forms colorless univalent ions, cadmium divalent, etc. These properties and their explanation are so nearly like those of the first long period that we need not consider them in more detail.

\section{The "Rare Earth" Period.}

After xenon the fourth shell begins. 'There are 32 cells to be filled by electrons before the atom again reaches the stability of an inert gas (niton). The first 3 or 4 elements have predominantly electropositive character and form positive ions whose valency increases by steps of one due to the tendency to revert to xenon. As more electrons are added they will tend, according to Postulate 6 , to arrange themselves over the I 8 of the underlying third shell, just as in the first long period the electrons arranged themselves over the 8 underlying ones in the second shell. This process yields a series of similar elements having about the same valence, just as we found among the elements from titanium to nickel: By referring to Table I we see that this farmily of elements corresponds exactly with the rare earth elements. The eighteenth element from xenon is lutecium, and this marks definitely the last of the rare earths.

Since the forces holding these 18 electrons are predominantly magnetic and since the constraints are not of the rigid kind characteristic of the inert gases, we should expect these elements to be paramagnetic. As a matter of fact, the rare earths are the most strongly paramagnetic of any of the elements except those from manganese to nickel. Even barium begins to show a perceptible paramagnetism (strontium is diamagnetic). The susceptibilities of only a few of these metals have been determined but the atomic susceptibilities of cerium, praseodymium, neodymium and erbium are, respectively, 2, 7, II and 7 times that of manganese. Gadolinium sulfate lies between ferric sulfate and manganese chloride in magnetic properties.

It seems probable that the most marked magnetic properties occur with the elements samarium, europium and gadolinium for these are the 
eighth, ninth and tenth elements from xenon and thus should correspond most closely in their structure to iron, nickel and cobalt. In samarium there is probably a slight tendency for the 8 electrons in the outside layer to arrange themselves at the corners of a cube, while in gadolinium the two extra electrons are in the polar axis. But in other properties these 3 elements should not differ radically from the other rare earths.

By the time the 18 electrons have been added the electrostatic forces have begun to oppose the magnetic attraction to a marked degree. Therefore, when in tantalum an additional electron is added, the whole outside shell tends to rearrange itself so that the empty cells will come opposite the electrons of the underlying shell. The most symmetrical arrangement of this kind will occur when there are 18 empty cells opposite the 18 underlying electrons. The atomic number of niton in which the fourth shell is complete is 86 -therefore an element having 18 empty spaces in the fourth will have an atomic number 68 corresponding to erbium. The structure of this $\beta$-form of erbium has the same kind of stability for large, nuclear charges that we found in the cases of $\beta$-nickel and $\beta$-palladium. We may therefore expect that the atoms beyond lutecium will show a marked tendency to revert to $\beta$-erbium. Thus tantalum with an atomic number 73 tends to lose 5 electrons and tungsten to lose 6 . The properties of tantalum and tungsten thus resemble those of columbium and molybdenum, but because of the complexity of the atom to which they revert, and in general because of the large numbers of electrons in their outside shell, their secondary valence forces are more highly developed.

In accordance with the marked change in the electron arrangement beyond lutecium we find that the paramagnetism is practically absent in the elements tantalum and tungsten.

The $\beta$-form of the erbium atom contains 18 empty cells arranged over the I 8 cells of the third shell. When electrons are added as we pass to elements of large atomic number the first 8 of them naturally tend to arrange themselves at the corners of a cube, because of the magnetic attraction of the 8 electrons in the second shell. The next two electrons for reasons of symmetry then arrange themselves in the polar axis. We thus have the 3 "eighth group" elements osmium, iridium and platinum. Because of the weakness of the forces acting between the fourth and the second shell we should not expect strongly developed magnetic properties in these elements. As a matter of fact osmium and iridium have susceptibilities nearly equal to zero, but there is a small but sharp rise at platinum making this element about $1 / 4$ as paramagnetic as palladium. The next elements gold, mercury, etc., are distinctly diamagnetic. The same sharp break occurs here as we found between nickel and copper, palladium and silver, lutecium and tantalum, although its magnitude is 
much less. We may therefore assume that beyond platinum the electrons tend to rearrange themselves in a $\beta$-form in which the ro electrons that have been added since erbium endeavor to get farther away from those of the underlying electrons. The 8 empty cells tend to take symmetrical positions in the atom probably at comers of a cube, and the cells containing electrons, space themselves as best they can. The fact that an arrangement of this kind does not have nearly the symmetry which we found for the $\beta$-form of the nickel atom is probably the explanation of the fact that the tendency of the succeeding elements to revert to this $\beta$-form of platinum is much less marked than we observed in the cases of reversion to nickel, palladium and erbium. Thus we find that gold and mercury have variable valence differing in this respect from silver and cadmium. Thallium forms univalent and tervalent ions whereas indium forms only tervalent. Lead only exceptionally is quadrivalent, while this seems to be the normal condition of tin compounds. Thus stannous salts are strong reducing agents, but divalent lead salts are not. Bismuth is normally tervalent and forms only a few very unstable compounds in which it is quinquivalent. Antimony on the other hand has about equal tendencies to be tervalent or quinquivalent.

There is an interesting sudden break in the susceptibility curve between lead and bismuth. Gold, mercury and thallium are very slightly diamagnetic, but bismuth is the most strongly diamagnetic element with the exception of the inert gases. In all of the elements between gold and niton the positions of the electrons are determined mainly by electrostatic forces (Postulate 6). But magnetic forces still tend to cause the electrons to arrange themselves in the 8 available cells (at platinum) so that they will be placed as symmetrically as possible with respect to the underlying electrons. Now the 4 additional electrons (in lead) can arrange themselves in the 8 spaces with reasonable symmetry, but the 5 electrons in bismuth cannot do so. The extra electron displaces the others and thus weakens the magnetic forces and strengthens the electrostatic. In agreement with this theory we find that there is a similar, although smaller, minimum in susceptibility at phosphorus, arsenic, and antimony, the elements which also have atomic numbers 3 less than those of the following inert gases. We also find distinct maxima at germanium, tin, and lead which have 4 electrons less than the inert gases which follow thern.

\section{The Uranium Period.}

With niton the first layer of electrons in the fourth shell is completed. As we add more electrons we should expect to go through the same cycle as that of the rare earth period. The properties of the first two or three elements are determined primarily by the ease with which they give up electrons. Thus radium very closely resembles barium, differing from 
it in chemical properties only by its slightly greater secondary valence which manifests itself here by slightly decreased solubility of its salts.

Thorium resembles cerium and zirconium, the elements of similar constitution.

Beyond thorium we might expect another series of elements analogous to the rare earths up to an atomic number of $\mathrm{IO}_{4}$ if the nuclei of such elements were stable enough to exist under ordinary conditions. However, the properties of uranium do not bear out this supposition. Uranium is not closely related in its properties to neodymium, nor in fact does it closely resemble any other element. It would seem therefore that it is not safe from our present knowledge to make definite predictions as to the properties of other possible elements of this period.

\section{The Mechanism of Chemical Action and the Structure and Properties of Compounds.}

The preceding theory of structure of the atoms in the two short periods is nearly the same as that postulated by Lewis. Lewis has disçussed how a theory of valence may be derived from this structure. He considers the nature of the single, double and triple bonds, the structure of the ammonium ion, iodine and oxygen molecules, ions such as sulfate perchlorate, etc., the tetrahedral arrangement of the electrons around the nucleus in the carbon atoms, etc. In this way he has fully demonstrated the general value and applicability of the theory. Unfortunately Lewis' treatment of this subject was rather brief and perhaps for this reason it does not seem to have met with the general acceptance which it deserves.

It therefore seems desirable to consider in some detail how this theory may be applied to the prediction of the properties of the first 18 elements and their compounds. In doing so we shall considerably extend Lewis' theory and because of the more definite conceptions of atomic structure which we have developed we will be able to apply a somewhat different viewpoint. In particular we shall attempt to explain the "physical" as well as the "chemical" properties of compounds. The predictions of these properties depends mainly on Postulates 8 , 9, io and I I given below. Following Lewis' practice we will refer to the outside electrons forming an uncompleted shell or layer as "the shell" of the atom, while the whole inner portion, consisting of shells and layers each containing its full quota of electrons, will be called the kernel.

Postulate 8.-The very stable arrangements of electrons corresponding to those of the inert gases are characterized by strong internal but unusually weak external fields of force. The magnetic and electrostatic forces are each very nearly internally balanced. The smaller the atomic number of the element the weaker are these external fields. 
Postulate 9.-The pair of electrons in the helium atom represents the most stable possible arrangement. A stable pair of this kind forms only under the direct influence of positive charges.

The positive charges producing the stable pair may be:

(a) The nucleus of any element.

(b) Two hydrogen nuclei.

(c) A hydrogen nucleus together with the kernel of an atom.

(d) T'wo atomic kerneis.

These are listed in the order of their stability.

As we have seen from the previous discussion on atomic structure, the tendency to form a pair of electrons about the nucleus of any atom over"powers the tendency to form other arrangements so that the stable pair constitutes the first shell of all the elements except hydrogen. The tendency for the pair to form around two atomic kernels (Case $d$ ) is weak and thus only in exceptional cases, under some outside compelling influence does this combination occur.

Postulate I0.--After the very stable pairs (Postulate 9) the next most stable arrangement of electrons is the group of 8 such as forms the outside layer in atoms of neon and argon. We shall call this stable group of 8 electrons the "octet." Any atom up to argon having more than two positive charges on its kernel tends to take up electrons to form an octet. The greater the charge on the kernel the stronger is this tendency. In exceptional cases, the octet can form about a complex kernel, that is, about a structure containing the kernels of two atoms bound together by a pair of electrons (Postulate $g d$ ).

Postulate II, Electrons Held in Common.-Two octets may hold I, 2, or sometimes even 3 pairs of electrons in common. A stable pair and an octet may hold a pair of electrons in common. An octet may share an even number of its electrons with $\mathrm{I}, 2,3$, or 4 other octets. No electrons can form parts of more than two octets.

The fact that only an even number of electrons can be held in common probably signifies that the tendency to form stable pairs between the two kernels according to Postulate 9 is a vital factor in the sharing of electrons between octets.

When octets combine together by sharing their electrons fewer electrons are required than if the octets remain separate. Thus when two octets containing a total of 16 electrons combine so as to hold a pair in common, two electrons are set free. Two octets held together by one, two, or three pairs of electrons thus contain $\mathrm{I}_{4}, \mathrm{I}_{2}$, or Io electrons, respectively. When we consider that the shells of all atoms except those of the inert gases are unsaturated, we see how necessary it is for the atoms to share their electrons with each other if the stable pairs and octet are to be formed. 
Lewis discusses two possible arrangements of the electrons in the octet. They may be placed at the 8 corners of a cube or they may be located in pairs at the 4 corners of a regular tetrahedron. In view of Postulates 5 and II it would seem that the electrons in the octet are normally arranged in positions corresponding to the corners of a cube but that the electrons which are held in common between two octets or an octet and a stable pair, are drawn together by magnetic forces to form pairs. Thus we look upon electrons in the atoms as able to move from their normal positions under the influence of magnetic and electrostatic forces. This view does not conflict with that of Postulate 3 according to which the electrons are in cellular spaces. Thus we might assume when there are 8 outside cells that these are in the form of octants of a sphere. In the atom of neon, or the kernel of sodium, or in the chlorine ion the 8 electrons would be arranged nearly at the centers of the octants, but in compounds where pairs of electrons are shared by other atoms, the two electrons forming the pairs are displaced over near the boundaries of the octants and are thus able to come sufficiently close together for the magnetic forces to cause them to form stable pairs.

We are now in a position to apply the theory to explain the properties of the elements and their compounds.

Hydrogen $N=\mathrm{I} ; E=\mathrm{I} .^{1-}$-Hydrogen atoms are very active chemically because they tend to combine with any other atoms capable of supplying electrons by which the stable pairs can be formed. We should expect a hydrogen atom to constitute a doublet of high moment which would tend to attract all other bodies. Thus atomic hydrogen is very strongly adsorbed on surfaces. ${ }^{2}$ When two hydrogen atoms come in contact their two electrons form a stable pair (Postulate $9 b$ ) under the influence of the two nuclei so that a molecule, $\mathrm{H}_{2}$, is produced. This molecule has an unusually weak external field (Postulate 8 ) and therefore hydrogen has a very low boiling point ${ }^{3}$ and is relatively inert chemically except in so far as it can be made to split up into atoms.

Helium $N=2 ; E=0$.- In the helium atom the stable pair already exists. Since this is the most stable arrangement of electrons and has the weakest stray field (Postulate 8), helium forms no chemical compounds and has the lowest boiling point and highest ionizing potential of any known substance. Hydrogen has a higher boiling point and lower ionizing

${ }^{1}$ We will use $E$ to denote the number of electrons in the shell of the atom as given in Table $I$.

2 Langmuir, This Journal, 34, I3 10 (I9I2).

3 According to the theory which I have advanced (ThIs JourNaL, 38, 222 (1916); 39, I 848 (I $\mathrm{I}$ I 7 )), the so-called physical properties such as boiling point, melting point, surface tension, etc., are manifestations of typical chemical forces, sometimes primary valence, other times secondary valence forces. 
potential than helium because the mutual repulsion of the two nuclei forces these apart and increases the strength of the external field.

\section{First Short Period.}

Lithium $N=3 ; E=$ I. - Two of the electrons form a stable pair which completes the first shell. The extra electron, just as does that of the hydrogen atom, tends to make the atom very active chemically. Lithium atoms are electric doublets and therefore attract one another. There is however now little or no tendency to form stable pairs of electrons (Postulate 9) since the kernels of the lithium atoms are not simple nuclei as were those of hydrogen. Thus after one lithium atom has drawn another one to it there is still just as great a force tending to draw in a third. The electrostatic forces involved are just like those holding together a crystal of sodium chloride. The positively charged lithium kernels and the free electrons will therefore arrange themselves in space in a continuous lattice in a manner quite analogous to that of sodium and chlorine atoms in crystals of sodium chloride. Lithium atoms when allowed to come into contact with each other do not form molecules but form a crystalline solid containing free electrons as part of the lattice structure which is therefore a metallic conductor of electricity. When lithium is melted the structure is not essentially changed except in regard to the regularity of the lattice. The free electrons still occur between the lithium kernels, and the metallic conduction persists. The strong forces exerted by the positively and negatively charged particles on each other makes it difficult to separate the atoms. The great difference between the boiling points of lithium and those of hydrogen and helium is understandable.

When lithium is heated to a sufficiently high temperature, the thermal agitation is able to overcome in some degree even these strong forces so that the lithium evaporates. It is easy to see however that the vapor is monatomic. The energy necessary to separate two atoms of lithium from the surface is about twice that required to separate one, but the kinetic energy of a molecule of two atoms is the same as that of one, so that the momentum is only $\sqrt{2}$ times that of a single atom. Or to look at the problem another way, suppose that a diatomic molecule of lithium, $\mathrm{Li}_{2}$, does evaporate from the surface, the kinetic energy of agitation of these atoms with respect to each other is the same as when the atoms formed part of the surface. But the forces holding the atoms together in space are in general much less than those which originally held the atoms to the surface. Thus if we assume that the space lattice is like that of sodium chloride each charged particle in the interior has 6 oppositely charged particles around it. A particle in the surface usually has two or three neighboring oppositely charged particles. For these teasons even if some diatomic lithium molecules should leave the surface 
they would dissociate into atoms at a rate, large compared to that at which they evaporate from the surface. In any case the resultant vapor is monatomic.

The attractive forces between the electrons and the lithium kernels prevent their separation and hence lithium vapor is normally a nonconductor of electricity.

If lithium atoms and hydrogen atoms are brought together the extra electron of the lithium atom and the electron of the hydrogen atom combine together to form a stable pair with the hydrogen nucleus at its center (Postulate 9a). The lithium kernels thus become lithium ions, $\mathrm{Li}^{+}$, while the hydrogen nuclei surrounded by the pair of electrons are negatively charged hydrogen ions, $\mathrm{H}^{-}$. These charged particles would be attracted to each other but since there is no tendency for negative ions to form pairs about positive kernels there would be no tendency to form molecules. The lithium and hydrogen ions form a crystalline solid having the composition LiH. Since there are no free electrons, the solid body is a non-conductor of electricity. If melted, however, the positively and negatively charged particles should be able to move under the influence of an electric field so that molten LiH should be an electrolyte (as Lewis has pointed out) in which hydrogen should appear at the anode. The comparative ease with which an electron can be taken from a lithium atom by an electronegative element makes univalent lithium ions stable in water solutions.

The theory thus not only accounts for the chemical activity and valence of hydrogen and lithium as compared to helium, but explains the ordinary properties, such as boiling point, electric conductivity, ionizing potential, etc.

Beryllium $N=4 ; E=2$. - The first two electrons form the stable pair, leaving two electrons in the second shell. Since the atom can give up two electrons easily, it forms a divalent ion.

Boron $N=5 ; E=3$.-The 3 electrons in the outer shell give this element its tervalent character. The small volume of the atom makes it incapable of forming a tervalent cation. Boron has therefore a more electronegative character than the previously considered elements.

Carbon $N=6 ; E=4$; Nitrogen $N=7 ; E=5 ;$ Oxygen $N=8$; $E=6$.-We shall consider these 3 elements together because the application of the theory is best illustrated by the compounds they form with each other and with hydrogen. The properties of the atoms up to this point have been determined by their ability to give up one or more electrons. With carbon and the elements which follow it there is less tendency to part with electrons, and more tendency to take up electrons to form a new octet. This opens up new possibilities in the formation of compounds and as a result we find a remarkable contrast between the prop- 
erties of oxygen and nitrogen and those of lithium and beryllium. The ordinary theory of valence has nowhere been more strikingly useful than in the chemistry of carbon compounds. Among compounds of carbon with hydrogen and oxygen the valence almost without exception can be taken as four for carbon, two for oxygen and one for hydrogen. This simple theory makes it possible to predict with certainty the existence of great numbers of compounds and the non-existence of others.

When nitrogen is introduced into organic compounds there is often much more uncertainty in using this theory of valence. But among the compounds of nitrogen with oxygen the same theory is almost useless. Who, for example, would ever have been able to predict the existence of such compounds as are represented by the formulas $\mathrm{N}_{2} \mathrm{O}, \mathrm{NO}, \mathrm{NO}_{2}$, $\mathrm{N}_{2} \mathrm{O}_{4}, \mathrm{~N}_{2} \mathrm{O}_{3}$ and $\mathrm{N}_{2} \mathrm{O}_{5}$ or $\mathrm{HNO}, \mathrm{HNO}_{2}, \mathrm{HNO}_{3}$, etc., by applying the valence theory that has been so successful in organic chemistry? But because of its great success in its special field this theory has been nearly universally used even for inorganic compounds. To explain the existence of the above oxides of nitrogen it has thus been assumed that the valence of nitrogen may be one, two, 3,4 or 5 . It is obvious that such a theory must predict the existence of an unlimited number of compounds which do not exist at all. For example, we should have such compounds as are indicated by the formulas $\mathrm{NH}, \mathrm{NH}_{2}, \mathrm{NH}_{4}$, or $\mathrm{NCl}, \mathrm{NCl}_{2}, \mathrm{NCl}_{4}$ and $\mathrm{NCl}_{5}$.

The degree to which any given theory of atomic structure is able to explain the success of the ordinary valence theory for carbon compounds and its failure for nitrogen compounds should serve as a measure of the value of the theory and should afford information as to whether the theory corresponds to the actual structure of the atoms.

With the exception of compounds like lithitum hydride and some compounds of elements having atomic numbers greater than 20 , nearly all "primary valence" compounds involve the formation of octets. Let us examine more closely the theory of valence which results from the application of Postulates 9, ro and II.

\section{Octet Theory of Valence.}

Let $e$ be the total number of available electrons in the shells of the atoms forming a given molecule. Let $n$ be the number of octets formed by their combination and let $p$ be the number of pairs of electrons held in common by the octets. For every pair of electrons held in common there is a saving of $2 p$ in the number of electrons needed to form the octets. Thus we have

$$
e=8 n-2 p .
$$

For most purposes it is more convenient to use this equation in the form

$$
p=1 / 2(8 n-e) .
$$


When a hydrogen nucleus holds a pair of electrons in common with an octet, this pair should not be counted in determining the value of $p$, since it does not result in any saving in the numbers of electrons required to form the octets.

To determine $e$ we add together the numbers of available electrons in the outside shells of all the constituent atoms. Thus for every hydrogen we add one, for lithium one, for beryllium 2, for carbon 4 , nitrogen 5 , and oxygen 6 .

Equation 2 gives definite information as to the ways in which the octets in a given molecule can be arranged. This equation applies to all "octet compounds," that is, to all compounds whose atoms are held together either because their octets share electrons or because electrons have passed from one atom to another in order to complete the octets.

Let us now apply Equation 2 to determine the structure of various molecules.

Water $\left(\mathrm{H}_{2} \mathrm{O}\right)$. -The hydrogen nuclei always tend to hold pairs of electrons, never octets. We thus place $n=\mathrm{I}, e=8$ ( 6 for the oxygen and one for each hydrogen). Whence, by Equation 2, $p=0$. This means that no electrons are held in common between octets, which must obviously be the case for any molecule containing only one octet. The fact that we found $p=0$ thus shows that the compound $\mathrm{H}_{2} \mathrm{O}$ can exist. The two hydrogen nuclei attach themselves to two pairs of electrons forming the octet. The arrangement is presumably as illustrated in Fig. 2. The hydrogen nuclei are represented by the + signs and

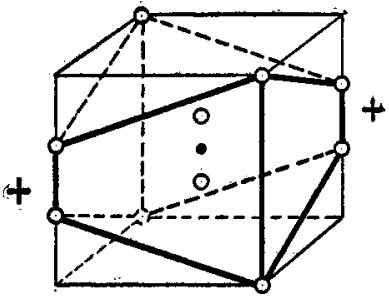

Fig. 2.

Diagram of the water molecule. the electrons in the octet by the small circles while the nucleus of the oxygen atom is shown as a black circle. In figures after this one the kernels of the atoms will not be shown.

We see from this structure that water forms molecules which are quite thoroughly saturated. The two hydrogen nuclei are held firmly by the pairs of electrons very much as they are in gaseous hydrogen. All the electrons form an octet and hence (Postulate 8) should have a rather weak stray field of force. Water therefore should be a substance easily volatile as $\mathrm{H}_{2} \mathrm{O}$ molecules and should not be a good conductor of electricity in the liquid state. Because of the lack of symmetry of the molecule as compared to the neon atom, water molecules should have a larger external field than neon atoms so that the boiling point should be much higher.

Lithium Oxide, $\mathrm{Li}_{2} \mathrm{O}$. - As before we place $n=\mathrm{I}, e=8$ and find $p=0$. The oxygen atoms have completed their octets by taking the two electrons from the two lithium atoms. The lithium kernels however already have 
their pairs of electrons and therefore cannot form pairs with those of the oxygen octet. Therefore lithium oxide consists of oxygen atoms carrying a double negative charge and of lithium kernels with single positive charge. This substance thus tends to form a solid space lattice structure having low vapor pressure, which is an electrolytic conductor when melted.

Lithium Hydroxide, $\mathrm{LiOH}, n=\mathrm{I}, e=8, p=0$.--The hydrogen nuclei are held by a pair of electrons in the oxygen octet but the lithium kernel does not share electron with the oxygen atom. This substance should thus form a solid body showing electrolytic conductivity when molten and capable of dissolving in water as an electrolyte giving ions of $\mathrm{Li}^{+}$and $\mathrm{OH}^{-}$. We should expect lithium hydroxide to be easily soluble in water because the $\mathrm{OH}^{-}$ion so much resembles water in its structure and the high dielectric constant of water makes it easy for the necessary separation of the positive and negative particles to take place.

Carbon Dioxide, $\mathrm{CO}_{2}$.- - Here each atom forms an octet. We place $n=3: e=4+2 \times 6=16$, whence, by Equation 2, $p=4$. Thus 4 pairs of electrons must be held in common by the 3 octets. This leads to a structure like that shown diagrammatically in Fig. 3 or probably better by Fig. 4 in which the pairs of electrons held in common are shown

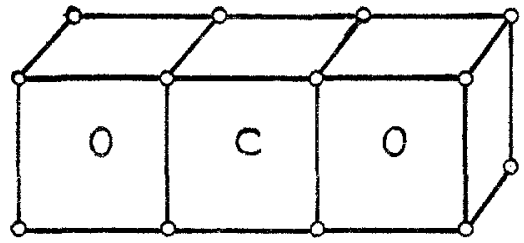

Fig. 3.

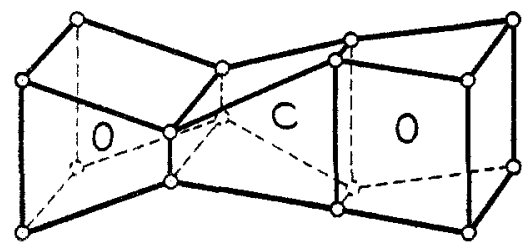

Fig. 4.

Figs 3 and 4,-Diagrams of carbon dioxide molecules.

closer together. This places the 4 pairs of electrons forming the octet of the carbon atom at the 4 corners of a regular tetrahedron. The lines connecting the electrons in these figures are used merely to give a better perspective of the arrangement of the electrons and of course should not be taken as representing the boundaries of the atoms. In the remainder of the figures no attempt will be made to show the probable closer approach of the electrons forming the pairs, that is, we will use only diagrammatic figures like Fig. 3 .

We can readily see that carbon dioxide is a thoroughly saturated nonpolar substance which should be a non-conductor of electricity, be very readily volatile and rather inert chemically. The structure of carbon dioxide given by our theory is in full accord with that given by the ordinary valence theory $\mathrm{O}=\mathrm{C}=\mathrm{O}$. By Fig. 3 we see that each pair of electrons held in common is the equivalent of the bond of the ordinary theory. 
The same was true of the structure found for water. But for lithium oxide and hydroxide there are no electrons held in common; the number of electrons transferred from one atom to the other corresponds to the ordinary valence.

Methane, $\mathrm{CH}_{4}$.-We place $n=\mathrm{I}, e=8$; hence $p=0$. The 4 hydrogen atoms supply 4 electrons to complete the octet of the carbon atom. Each hydrogen nucleus is held by one of the 4 pairs of electrons forming the octet. The final arrangement is that the 4 hydrogen atoms are located at the corners of a tetrahedron, each atom held by a stable pair just as it is in the hydrogen molecule. This arrangement is very much more symmetrical than that of the water molecule and should have a much weaker external field. Methane should thus be non-polar to an unusual degree; it should be a gas with a fairly low boiling point, and should be a non-conductor even when liquid.

Organic Compounds.- It will be found that Equation 2 leads to results identical with those of the ordinary theory of valence for all organic compounds of carbon, oxygen and hydrogen. In every case each pair of electrons held in common corresponds to a bond. Two and 3 pairs held between two octets correspond to the double and triple bonds respectively.

To show how this theory applies to hydrocarbons let us attempt to find according to the octet theory what substances of the general formula $\mathrm{C}_{x} \mathrm{H}_{y}$ can exist.

From Equation 2, it is evident that in all octet compounds there must be an even number of electrons $e$, otherwise $p$, the number of pairs of electrons held in common, would be a fractional number. Therefore in all hydrocarbons there must be an even number of hydrogen atoms since each of these atoms has a single electron. We may therefore confine our at-

TABLE II.

\begin{tabular}{|c|c|c|c|c|}
\hline & $n$. & e. & D. & Constitution. \\
\hline $\mathrm{CH}_{2}$ & I & 6 & $\mathbf{I}$ & Impossible \\
\hline $\mathrm{CH}_{4}$ & I & 8 & o & $\mathrm{CH}_{4}$ \\
\hline $\mathrm{CH}_{6}$ & I & ro & -1 & Impossible \\
\hline $\mathrm{C}_{2} \mathrm{H}_{2}$ & 2 & Io & 3 & $\mathrm{HC} \equiv \mathrm{CH}$ \\
\hline $\mathrm{C}_{2} \mathrm{H}_{4}$ & 2 & 12 & 2 & $\mathrm{H}_{2} \mathrm{C}=\mathrm{CH}_{2}$ \\
\hline $\mathrm{C}_{2} \mathrm{H}_{6}$ & 2 & I4 & I & $\mathrm{H}_{3} \mathrm{C}-\mathrm{CH}_{3}$ \\
\hline $\mathrm{C}_{2} \mathrm{H}_{8}$ & 2 & 16 & $\circ$ & $\mathrm{CH}_{4}+\mathrm{CH}_{4}$ \\
\hline $\mathrm{C}_{8} \mathrm{H}_{2}$ & 3 & 14 & 5 & Possible only in ring \\
\hline $\mathrm{C}_{3} \mathrm{H}_{4}$ & 3 & I6 & 4 & $\mathrm{H}_{2} \mathrm{C}=\underset{\mathrm{H}}{\mathrm{C}}=\mathrm{CH}_{2}$ \\
\hline $\mathrm{C}_{8} \mathrm{H}_{6}$ & 3 & 18 & 3 & $\mathrm{H}_{3} \mathrm{C}-\mathrm{C}=\mathrm{CH}_{2}$ or as ring \\
\hline $\mathrm{C}_{3} \mathrm{H}_{8}$ & 3 & 20 & 2 & $\mathrm{H}_{3} \mathrm{C}-\mathrm{CH}_{2}-\mathrm{CH}_{3}$ \\
\hline $\mathrm{C}_{3} \mathrm{H}_{1 \mathrm{C}}$ & 3 & 22 & I & $\mathrm{H}_{3} \mathrm{C}-\mathrm{CH}_{\mathrm{a}}+\mathrm{CH}_{4}$ \\
\hline $\mathrm{C}_{4} \mathrm{H}_{2}$ & 4 & 18 & 7 & $\mathrm{HC} \equiv \mathrm{C}-\mathrm{C} \equiv \mathrm{CH}$ \\
\hline $\mathrm{C}_{4} \mathrm{H}_{4}$ & 4 & 20 & 6 & $\begin{array}{l}\mathrm{H}_{2} \mathrm{C}=\mathrm{C}=\mathrm{C}=\mathrm{CH}_{2} \\
\text { ring }\end{array}$ \\
\hline
\end{tabular}


tention to hydrocarbons of the general formula $\mathrm{C}_{x} \mathrm{H}_{2 y}$. In Table II a series of compounds of this type is analyzed by the octet theory. The first column gives the empirical formula of the hypothetical compound. The values of $n$, and $e$ are in the next two columns while $p$ calculated by Equation 2 is in the fourth. For $\mathrm{CH}_{2}, p$ is found to be $\mathrm{I}$. Since a single octet cannot share any electrons with itself it is impossible to form a compound $\mathrm{CH}_{2}$. In the case of $\mathrm{CH}_{4}$ we find $p=0$ which is easily realized since there is only one carbon atom. Therefore the compound $\mathrm{CH}_{4}$ should exist. The compound $\mathrm{CH}_{6}$ is obviously impossible since for this the theory gives $p=-\mathrm{I}$. For $\mathrm{C}_{2} \mathrm{H}_{2}$ the equation gives $p=3$. The carbon atoms must therefore have 3 pairs of electrons in common. If we represent each pair by the line used to indicate a valence bond, the carbon atoms are represented by $\mathrm{C} \equiv \mathrm{C}$. There is no question as to where the hydrogen atoms must go. It is impossible to have $\mathrm{C} \equiv \mathrm{CH}_{2}$ because such a structure would require five pairs of electrons around one carbon atom. The formula therefore must be $\mathrm{HC} \equiv \mathrm{CH}$. In a similar manner we arrive at each of the other formulas. The only cases where the pairs of electrons can be shared between the octets of the carbon atoms in more than one way are the cases where isomers should exist according to the ordinary valence theory. Once having decided where the pairs of electrons are located, there is never any possibility of arranging the hydrogen atoms in any other than one way. The theory applies exactly as well to ring compounds as to chains.

The Equation 2 is thus a complete mathematical statement of the valence laws for hydrocarbons.

For carbon-oxygen-hydrogen compounds the theory works out in about the same way. All the structures given by the ordinary theory can be found by the octet theory and in every case each bond corresponds to a pair of electrons. An illustration of a compound of this type will help to make this clear.

Acetic Acid, $\mathrm{C}_{2} \mathrm{H}_{4} \mathrm{O}_{2}$. - Here $n=4, e=2 \times 4+4+2 \times 6=24$; whence $p=4$. The 4 pairs of electrons may be distributed in several ways such as $\mathrm{C}=\mathrm{C}<_{\mathrm{O}}^{\mathrm{O}}, \mathrm{C}-\mathrm{O}-\mathrm{C}=\mathrm{O}, \mathrm{C}-\mathrm{C} \mathrm{O}_{\mathrm{O}}^{\mathrm{O}}$, etc. ${ }^{1}$ These correspond to the compounds $\mathrm{H}_{2} \mathrm{C}=\mathrm{C}\left\langle\mathrm{OH}_{3}, \mathrm{H}_{3} \mathrm{C}-\mathrm{O}-\mathrm{CH}=\mathrm{O}\right.$ and $\mathrm{H}_{3} \mathrm{C}-\mathrm{C}_{\mathrm{OH}}^{\mathrm{O}}$ the last one being acetic acid.

${ }_{1}$ As far as the application of Equation 2 is concerned we might also arrange the pairs of electrons thus: $\mathrm{O}-\mathrm{C}=\mathrm{O}-\mathrm{C}$ corresponding to a compound, $\mathrm{O}-\mathrm{CH}=$ $\mathrm{O}-\mathrm{CH}_{3}$. It is only by taking into account the fact, to be discussed later, that oxygen has a tendency to share either one or two pairs of electrons and only rarely shares 3 pairs, that we can predict that a compound of the above type does not exist. With sulfur, compounds of this general type frequently do exist. 
Thus if we apply the octet theory to a compound $\mathrm{C}_{2} \mathrm{H}_{4} \mathrm{O}_{2}$ we find the same isomers that we would be led to by the ordinary valence theory. The ordinary valence theory sometimes indicates the possibility of compounds which we have never been able to prepare as for instance $\mathrm{C}(\mathrm{OH})_{4}$. For organic compounds the octet theory will be no better than the ordinary valence theory in this respect-the results will always be identical. But we shall see that this identity disappears in the case of inorganic compounds.

Oxides of Nitrogen.-There is hardly a case where the ordinary valence theory fails so completely as when it is applied to the oxides of nitrogen. Let us try to deduce from the octet theory what oxides of nitrogen might be capable of existence and what the structure of their molecules must be. In the first place from Equation 2 we see that the total number of available electrons in the molecule must be even. Since nitrogen has 5 electrons in its shell and oxygen has 6 , there will always be an odd number of electrons, unless the number of nitrogen atoms is even. We will therefore apply our theory to investigate the structure of the series of oxides represented by $\mathrm{N}_{2} \mathrm{O}_{x}$ as given in Table III.

\begin{tabular}{|c|c|c|c|c|c|}
\hline & & & TABL & III.- & RUCTURE OF NITROGEN OXIDES. \\
\hline & & $n$. & e. & p. & Structural formulas. \\
\hline I & $\mathrm{N}_{2} \mathrm{O}$ & 3 & 16 & 4 & $\mathrm{~N}=\mathrm{O}=\mathrm{N}$, or $\mathrm{N}=\mathrm{N}=\mathrm{O}$ \\
\hline 2 & $\mathrm{~N}_{2} \mathrm{O}_{9}$ & 4 & 22 & 5 & $O=N-N=0$ \\
\hline 3 & $\mathrm{~N}_{2} \mathrm{O}_{3}$ & 5 & 28 & 6 & $\begin{array}{l}O= \\
O\end{array}-O-N=O$, or $O=N-N=0$ \\
\hline 4 & $\mathrm{~N}_{2} \mathrm{O}_{4}$ & 6 & 34 & 7 & $O=\begin{array}{c}N-N=0 \\
O\end{array}$ \\
\hline 5 & $\mathrm{~N}_{2} \mathrm{O}_{5}$ & 7 & 40 & 8 & $\mathrm{O}=\mathrm{N}-\mathrm{O}-\mathrm{N}=\mathrm{O}$ \\
\hline 6 & $\mathrm{~N}_{2} \mathrm{O}_{8}$ & 8 & 46 & 9 & Impossible \\
\hline 7 & $\mathrm{~N}_{2} \mathrm{O}_{7}$ & 9 & 52 & IO & Impossible \\
\hline
\end{tabular}

The values of $p$ calculated from Equation 2 are given in the fifth column. The most probable structural formulas based on these values of $p$ are given in the last column. In these formulas, as throughout the rest of this paper, each dash represents a pair of electrons held in common between adjacent atoms, just as in the formulas of organic compounds.

When $x=\mathrm{I}$ we find that our theory indicates that such a compound has the formula $\mathrm{N}=\mathrm{O}=\mathrm{N}$ or $\mathrm{N}=\mathrm{N}=\mathrm{O}$. Because of its simplicity and symmetry we should expect such a compound to exist. The actual arrangement of the electrons is shown diagrammatically in Fig. $5 .{ }^{1}$ According to the octet theory there is no difficulty at all in

1 There is good evidence that nitrous oxide has the structure $\mathrm{N}=\mathrm{N}=\mathrm{O}$ rather than that shown in Fig. 5. The arrangement of the electrons however remains the same. 
explaining the existence and properties of this substance. Its structure is exactly like that of carbon dioxide (Fig. 3). We should thus expect it to be a gas having about the same boiling point as carbon dioxide. Its oxidizing properties at high temperatures are due to its decomposition into oxygen and nitrogen which is made an irreversible process by the great and unusual stability of the nitrogen molecule.

Let us proceed with the other oxides of nitrogen. When $x=2$ (Table III) we find that a possible arrangement is $O=N-N=O$. This formula is identical to that which we obtain by the ordinary theory if

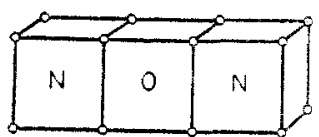

Fig. 5 .

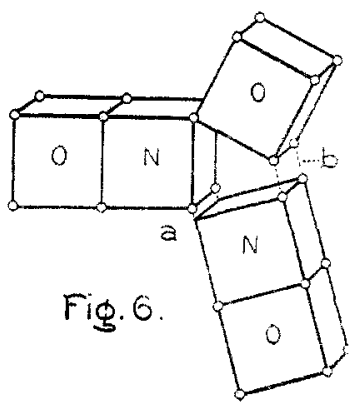

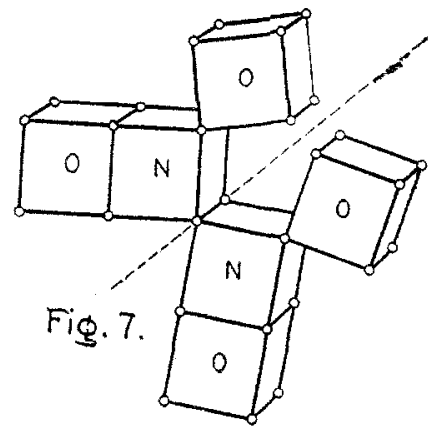

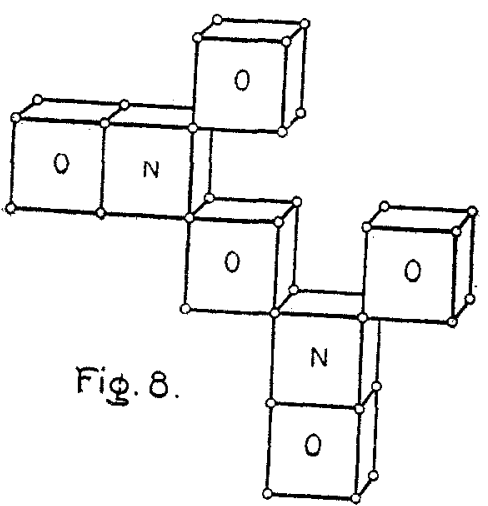

Figs. 5, 6, 7 and 8.-Diagrams of molecules of the oxides of nitrogen.

nitrogen is taken as tervalent. The fact that this compound does not exist as a gas, but presumably dissociates into NO is not explained by the octet theory but neither is it by the ordinary theory.

According to the octet theory $\mathrm{N}_{2} \mathrm{O}_{3}$ may have at least 3 different struc$\mathrm{O}$

tures $\mathrm{O}=\mathrm{N}-\mathrm{O}-\mathrm{N}=\mathrm{O}, \mathrm{O}-\mathrm{N}=\mathrm{O}=\mathrm{N}-\mathrm{O}$ or $\mathrm{O}=\mathrm{N}-\mathrm{N}=\mathrm{O}$ whereas by the ordinary theory with tervalent nitrogen-only the first should be possible. In view of the ease with which $\mathrm{N}_{2} \mathrm{O}_{3}$ breaks down to $\mathrm{NO}$ and $\mathrm{NO}_{2}$ and by comparison with the formula for $\mathrm{N}_{2} \mathrm{O}_{2}$ which breaks 
down into NO it seems that the third of the above formulas is the most probable structure. The deep blue color of liquid $\mathrm{N}_{2} \mathrm{O}_{3}$ indicates the presence of very loosely held electrons. This suggests tautomerism between the first and third forms as shown in Fig. 6 . If a pair of electrons is held in common at $a$ we have the third formula while if it is held at $b$ the structure is as given by the first formula. This tautomerism involves the shifting of two electrons between the positions $a$ and $b$.

The structure of $\mathrm{N}_{2} \mathrm{O}_{4}$ derived by the octet theory as given in Table III is as shown in Fig. 7. By comparing this with Fig. 6 we see that the extra oxygen atom has made the molecule incapable of tautomerism like that between the two forms of $\mathrm{N}_{2} \mathrm{O}_{3}$. On the other hand, the molecule still tends to dissociate into two parts $\left(\mathrm{NO}_{2}\right)$ as shown by the dotted line. The stability of the electrons in $\mathrm{N}_{2} \mathrm{O}_{4}$ causes this to be a colorless compound.

The octet theory also explains the existence of $\mathrm{N}_{2} \mathrm{O}_{5}$ and leads to the structure shown in Fig. 8. There are no unstable electrons so this compound is colorless. It tends to decompose as indicated by dotted lines into $\mathrm{NO}_{2}$ and oxygen, although not nearly as readily as does $\mathrm{N}_{2} \mathrm{O}_{4}$.

When we apply the octet theory to the case that $x=6$, or 7 we find (see Table III) that the value of $p$ becomes so large that there are not enough electrons in the atoms to form the pairs, except by forming chains of oxygen atoms or a ring structure. Even on the ordinary theory we could account for any numbers of oxygen atoms if we could string them out in chains thus as for example $\mathrm{O}=\mathrm{N}-\mathrm{O}-\mathrm{O}-\mathrm{N}=\mathrm{O}$. We see $\mathrm{O} O$

that such a structure as ONONO is impossible, since by Table III we $\mathrm{O} O$

would have $p=10$ and this would require more electrons in the shell of the nitrogen atom than there actually are. The octet theory thus explains without difficulty the existence and properties of $\mathrm{N}_{2} \mathrm{O}, \mathrm{N}_{2} \mathrm{O}_{3}$, $\mathrm{N}_{2} \mathrm{O}_{4}$ and $\mathrm{N}_{2} \mathrm{O}_{5}$, although the ordinary theory fails to do so. Both theories fail to explain the existence of $\mathrm{NO}$ and $\mathrm{NO}_{2}$. We shall see however that these compounds may be explained by a modification of the octet theory which we shall consider later.

Nitrogen Acids.- The structures of the three acids $\mathrm{HNO}, \mathrm{HNO}_{2}$ and $\mathrm{HNO}_{3}$ are given by the octet theory without the necessity of assuming variable valence. Thus for $\mathrm{HNO}, n=2, e=\mathrm{I} 2$ and $p=2$. This gives a structure represented by $\mathrm{HN}=\mathrm{O}$ or $\mathrm{HO}=\mathrm{N}$ and shown in Fig. 9. In a similar way we obtain the structures for $\mathrm{HNO}_{2}$ and $\mathrm{HNO}_{3}$ as given in Fig. 9. It should be noted that the group $\mathrm{O}=\mathrm{N}-\mathrm{O}$ which occurs in $\mathrm{HNO}_{3}$ is the same as in the formulas for $\mathrm{N}_{2} \mathrm{O}_{3}, \mathrm{~N}_{2} \mathrm{O}_{4}$ and $\mathrm{N}_{2} \mathrm{O}_{5}$. The group $\mathrm{O}=\mathrm{N}-\mathrm{O}-$ in $\mathrm{HNO}_{2}$ was previously found in the $b$-form 
of $\mathrm{N}_{2} \mathrm{O}_{3}$ shown in Fig. 6. We see that although $\mathrm{HNO}, \mathrm{HNO}_{2}, \mathrm{HNO}_{3}$ can exist, $\mathrm{HNO}_{4}$ cannot exist, for in this last case $p=5$ which would require to electrons around the nitrogen kernel.

Nitrogen-Hydrogen Compounds.--The octet theory indicates that $\mathrm{NH}_{3}$, and $\mathrm{H}_{2} \mathrm{~N}-\mathrm{NH}_{2}$ should exist and have the properties they do. The compounds $\mathrm{NH}, \mathrm{NH}_{2}, \mathrm{NH}_{4}$ and $\mathrm{NH}_{5}$ cannot exist since in these cases we find $p=I, 1 / 2,-1 / 2$, and $-I$ respectively, whereas $p$ must be equal to zero for a single octet. The theory ${ }_{0}$ thus automatically shows the radical difference between the number of oxygen and hydrogen compounds that may be formed. For the ammonium ion, $\mathrm{NH}_{4}$, we find $n=$ $x, e=8$ and $p=0$. The structure of this ion is thus exactly like that of the methane molecule. The positive charge is the cause of its resemblance to the potassium ion.

Hydronitric acid, $\mathrm{HN}_{3}$, gives $n=3 ; e=16$, and therefore $p=4$. This leads to the formula $\mathrm{HN}=\mathrm{N}=\mathrm{N}$ as the most probable structure.

Peroxides.- In the oxides of nitrogen and in the nitrogen acids we have seen how oxygen atoms can attach themselves to any octet in which
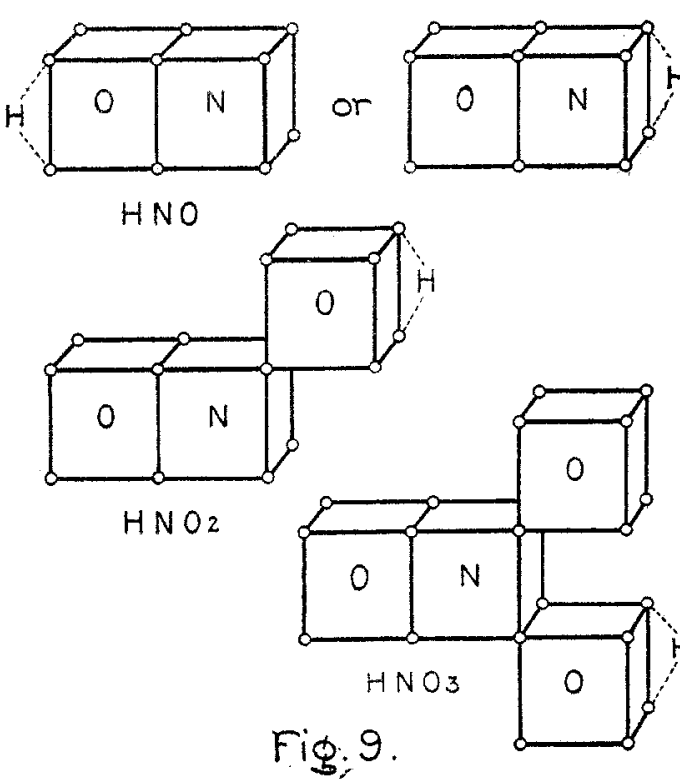

Diagrams of molecules of nitrogen oxy-acids. two or more of the electrons are not already held as stable pairs. Thus in Fig. 9, $\mathrm{HNO}_{3}$ is obtained merely by adding an oxygen atom to the only remaining free pair of electrons belonging to the nitrogen atom of $\mathrm{HNO}_{2}$. Since oxygen atoms have 6 electrons, they can form an octet by sharing two electrons with some atom which already has an octet. In the case of the nitrogen acids this process could go on until all of the electrons of the nitrogen octet were shared with oxygen atoms. This is quite analogous to the addition in organic molecules, of $\mathrm{CH}_{2}$ groups which also have 6 available electrons.

We shall see that oxygen atoms can thus add themselves not only to nitrogen atoms but to chlorine, bromine, iodine, phosphorus, sulfur, and other atoms. They can also add themselves to some extent to each other. Thus if we apply the octet theory to hydrogen peroxide, we find 
$p=\mathrm{I}$. This leads either to the structure $\mathrm{HO}-\mathrm{OH}$, or equally well to the structure $\mathrm{H}_{2} \mathrm{O}-\mathrm{O}$ as shown in Fig. Io.

As a matter of fact, the hydrogen nuclei are probably very mobile and readily shift from one pair of electrons in an octet to another. It may well be that the ease with which this shift occurs is the cause of the high dielectric constant of such substances as $\mathrm{H}_{2} \mathrm{O}, \mathrm{NH}_{3}$, etc. Such an effect does not exist in the case of the hydrogen in hydrocarbons, for all the electrons in the carbon octets are held as stable pairs so that the hydrogen atoms cannot shift positions. The structure given in Fig. $\mathrm{Iob}$ for $\mathrm{H}_{2} \mathrm{O}_{2}$ agrees well with most of its properties. According to the ordinary theory this structure implies a quadrivalent oxygen atom. But the
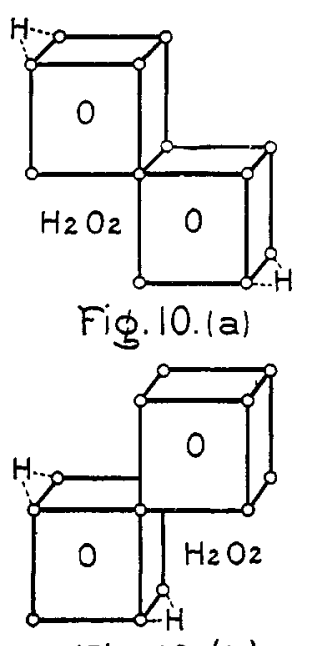

Fig. 10. (b)

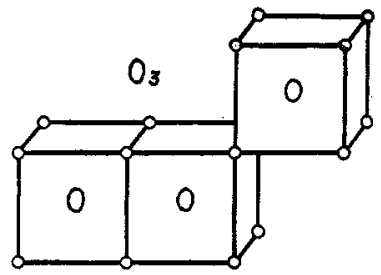

Fig.ll.(a)

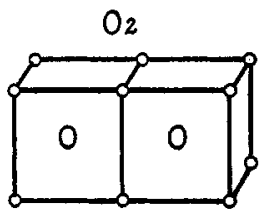

Fig. II.(b)

Diagrams of molecules of hydrogen peroxide and ozone. octet theory requires nothing at all unusual in the properties of the oxygen atom.

In a similar way for ozone we find $p=3$ which leads to the formula $\mathrm{O}=\mathrm{O}-\mathrm{O}$ as shown in Fig. I $\mathrm{r} a$. For the oxygen molecule, however, we find $p=2$ so the structure is $\mathrm{O}=\mathrm{O}$ as shown in Fig. $\mathrm{I} \mathrm{r} b$. This structure for ozone brings out its relationship to the peroxides much better than the ring structure suggested by the ordinary valence theory. The structure and symmetry of the oxygen molecule account for its stability and the low boiling point of liquid oxygen.

The Structure of the Nitrogen Molecule.-The properties of elementary nitrogen are in many ways extraordinary. The contrast between the properties of carbon and nitrogen, elements adjacent to each other in the Periodic Table, could hardly be more striking. Carbon does not melt even at $3700^{\circ} \mathrm{C}$. while nitrogen has a lower boiling point than any substance except hydrogen, helium and neon. Nitrogen, although many compounds with oxygen exist, combines with oxygen only at exceedingly high temperatures and even then only to a small degree. Carbon, notwithstanding the great affinity of its atoms for each other as shown by its low vapor pressure, combines readily with oxygen at moderate tempera- 
tures. Even at the temperature of melting tungsten (about $3600^{\circ} \mathrm{K}$.) there is evidence that nitrogen is not appreciably dissociated into atoms. ${ }^{1}$ This stability of the nitrogen molecule, which is so much greater than that of a compound such as carbon dioxide, must be due to some unusual kind of structure.

If we apply the octet theory to the nitrogen molecule by placing $n=2$; $e=$ Io we find $p=3$. We are thus led to the formula $\mathrm{N} \equiv \mathrm{N}$ for the nitrogen molecule. Now in acetylene we have an illustration of two atoms holding 3 pairs of electrons in common. Such a substance is endothermic, forms addition products easily, and even by itself is relatively unstable. A structure of this kind could not possibly account for the properties of nitrogen.

In its boiling point and in fact in most of its properties elementary nitrogen resembles argon. The boiling points of gases on the absolute scale, being approximately proportional to the molecular heats of evaporation, serve as a measure of the external field of force of the molecules. By examination of a large number of organic compounds it appears that the freezing point is dependent to a marked degree on the symmetry of the molecule - the more symmetrical the molecule, the higher is the freezing point. The following table gives the freezing points and boiling points of oxygen, nitrogen and argon on the absolute scale:

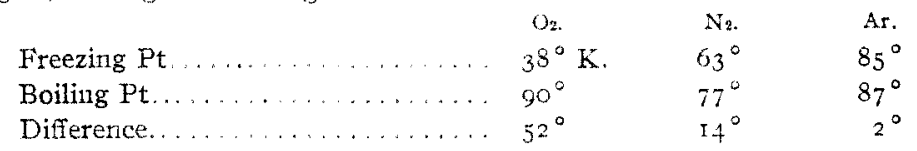

Judging from these boiling points the nitrogen molecule must have a weaker external field of force than either that of oxygen or argon. The differences between the freezing points and boiling points indicate that the nitrogen molecule is very much more symmetrical than that of oxygen and approaches that of argon. The outside layer of electrons in the argon atom consists of a single octet. The evidence given above suggests that the nitrogen molecule also has an outer shell consisting of a single octet. Is such an arrangement possible?

The two nitrogen atoms in a molecule have a total of $\mathrm{r}_{4}$ electrons. We may assume that each nucleus binds two electrons to form a stable pair according to Postulate 9. The two nitrogen kernels, each with 5 positive charges, are then surrounded by a total of ro electrons. There are thus two electrons more than are needed to form the octet. In view of the stability of the octet (Postulate 10) and the relatively large charges on the two kernels, it seems reasonable that the pair of extra electrons. should be imprisoned within the octet and be held by the two nitrogen kernels (Postulate $9 d$ ) as shown in Fig. 12.

' Langmuir, ThIs Journal, 34, 876 (IgI2). 
Structures of this kind are not usual but it is evident that in this case there are a number of exceptional factors operating to bring about just this result. The original nitrogen atoms are thoroughly unsaturated and very unsymmetrical and thus have large external fields of force. The number of electrons (5) in each shell is such that two atoms cannot form a molecule by forming two octets unless 3 pairs are held in common,

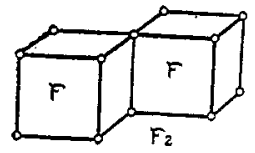

Fig. 13.

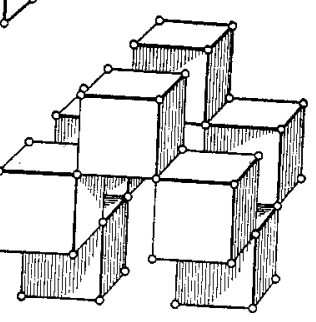

Fig. 17.
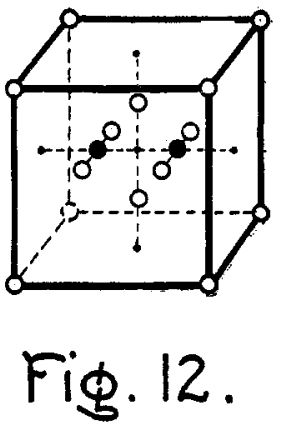

Fig, I 2.-Diagram of the Nitrogen Molecule.

Fig. 13.-Diagram of the Fluorine Molecule.

Fig. 1 7.-Diagram of the Sulfur Molecule $S_{8}$.

something which seldom occurs with nitrogen atoms. Three nitrogen atoms cannot combine to form a single molecule, for this would give an odd number of electrons. Finally, it so happens that there are only two electrons too many to form a complete single octet, and that there are two kernels of small volume but with large positive charges, to bind this extra pair.

This structure of the nitrogen molecule explains in a perfectly satisfactory way all the remarkable properties of elementary nitrogen previously mentioned. The high heat of formation of nitrogen molecules from the atoms accounts for the great number of endothermic and explosive nitrogen compounds. It is also evident why elementary nitrogen is so unusually inert, while in its compounds it is one of the most active of the elements.

Carbon Monoxide.-The structure of the molecule of this gas has long been a puzzling problem. According to the ordinary valence theory, carbon in this compound must be divalent, and hence should be very much unsaturated. Yet carbon monoxide is a relatively inert substance. Its very low boiling point proves that its molecules have very little external field of force. Its small solubility in water, and the difficulty of finding chemical substances which combine with it at room temperature, do not fit in well with the theory of the divalent carbon atom, 
for such an atom should show much more active properties-logically its properties should resemble those of atomic hydrogen.

As a matter of fact carbon monoxide resembles nitrogen to an extraordinary degree, as shown by Table IV, which indicates that the physical properties of these two gases are identical almost within the probable limits of experimental error.

\section{Table IV,-Physical Properties of Gases.}

\begin{tabular}{|c|c|c|c|c|c|}
\hline & co. & $\mathrm{N}_{2}$ & Ar. & $\mathrm{O}_{2}$ & No. \\
\hline Freezing point, degrees $\mathrm{K}$. & 66 & 63 & 85 & 38 & 106 \\
\hline Boiling point, degrees $K$.. & 83 & 78 & 87 & 90 & I20 \\
\hline Critical temp, degrees $\mathrm{K} \ldots \ldots \ldots \ldots$ & 122 & 127 & 156 & 155 & 180 \\
\hline Critical pressure, atm............ & 35 & 33 & 5.3 & 50 & 71 \\
\hline Critical volume $\ldots \ldots \ldots \ldots \ldots \ldots$ & 5.05 & 5.17 & 4.04 & 4.26 & $3 \cdot 5$ \\
\hline Solubility in water, $0^{\circ} \mathrm{C} .(\%) \ldots \ldots$ & $3 \cdot 5$ & 2.4 & 5.8 & 4.9 & $7 \cdot 4$ \\
\hline Density of liquid at boiling point. .... & 0.793 & 0.796 & $\ldots$ & $\ldots$ & \\
\hline Viscosity, $\eta \times 10^{6}$ at $0^{\circ} \mathrm{C} \ldots \ldots \ldots$ & 163 & $\mathrm{x} 66$ & 210 & 187 & 165 \\
\hline
\end{tabular}

This evidence alone should be sufficient to prove that the structure of the shell of the carbon monoxide and the nitrogen molecule must be nearly identical.

However, the case is still stronger when we consider that the total number of electrons in the molecule is the same in both gases. Thus in one case we have two nitrogen atoms with 7 electrons each, while in the other we have carbon with 6 and oxygen with 8 . Evidently the reasons which led us to assume that the nitrogen molecule has an outside shell, consisting of a single octet apply with the same force to the carbon monoxide molecule.

In the carbon monoxide molecule there is then an imprisoned pair of electrons within the octet held as a stable pair by the carbon and the oxygen kernels having charges of 4 and 6 , respectively.

This theory explains why nitrogen and carbon monoxide are alike in all properties in which a separation of the kernels is not involved. The fact that the two kernels have unequal charges makes it much easier for this structure to break down. Thus while nitrogen shows no signs of dissociating at temperatures of a couple of thousands of degrees, carbon monoxide enters into a few reactions even at room temperature and many reactions at temperatures of a few hundred degrees. Its reaction velocity is however usually very slow indicating that only a small fraction of the molecules is in a form in which the kernels can be separated.

Once the kernels have been separated the carbon monoxide behaves like a very much unsaturated body. It seems probable that at higher temperatures, the carbon monoxide exists in two tautomeric forms-one in which the outer shell is a single octet, and the second in which there are two octets. According to the octet theory the formula of this second 
form would be $\mathrm{C} \equiv \mathrm{O}$. This would immediately react with oxygen atoms to form $\mathrm{O}=\mathrm{C}=\mathrm{O}$.

Nitric Oxide.-Lewis has pointed out that among the first 20 elements there are only about a half dozen cases in which there is an odd number of electrons in a molecule. With the exception of nitric oxide, these are all substances which by their color or their intense chemical activity act as though they might have a free electron either in the outside shell or close to it. Nitric oxide however is colorless and very stable even at high temperatures. Although it reacts with oxygen to form nitrogen dioxide, this reaction takes place rather slowly, so that the claim can hardly be made that the substance has remarkable chemical activity. Compared to the other oxides of nitrogen it is characterized by its very low boiling point.

The molecule of this substance contains is electrons-just one more than the molecules of nitrogen or carbon monoxide. It seems most probable that it has a similar structure and that the extra electron is imprisoned within the octet comprising the shell. This process seems more probable when we remember that the two kernels of the nitric oxide molecule have a total of I I positive charges as compared to the ro in the nitrogen and carbon monoxide molecule. With this structure the odd electron is so placed that it does not produce the effects usually characteristic of an odd electron. It is of interest in this connection that nitric oxide is remarkably paramagnetic. This is undoubtedly dependent in some way on this internal odd electron.

According to the last column of Table IV the properties of nitric oxide differ very appreciably from those of nitrogen and carbon monoxide, but not more so than we should expect from the presence of an extra electron within the shell. All the differences in properties shown in this table indicate that the odd electron increases the external field of the atom. The greater chemical activity as compared to nitrogen and carbon monoxide is also due to the same cause.

Hydrocyanic Acid.- There has been much discussion as to the constitution of this compound. It is generally agreed, I think, at present that it has a divalent carbon atom like that in carbon monoxide and is represented by the formula $\mathrm{H}-\mathrm{N}=\mathrm{C}$.

The total number of electrons in this molecule is I $_{4}$-the same as in the nitrogen and carbon monoxide molecules. This fact together with its resemblances to carbon monoxide suggest that here again we have an imprisoned pair of electrons within an outside shell consisting of an octet. The total charges on the kernels of nitrogen and carbon is 9 instead of Io as in the nitrogen and carbon monoxide molecules. This somewhat decreases the stability and at the same time gives a negative charge to the $\mathrm{CN}$ radical so that it must combine with a hydrogen nucleus, or 
form a negative ion. Two such ions can combine by sharing a pair of electrons-thus releasing the electrons and leaving the uncharged cyanogen molecule $(\mathrm{CN})_{2}$. It is well known that cyanogen has a very close resemblance to chlorine in many ways. Thus chlorides and cyanides are often isomorphous; silver chloride and cyanide have similar solubilities, etc. The theory of the constitution given above indicates that this resemblance is not accidental, nor is it merely due to the fact that both ions are univalent. It depends on the fact that both the cyanogen ion and the chlorine ion have outside shells that consist of a single octet. The relation between these two ions is like that between elementary nitrogen and argon.

It seems that these 4 substances, nitrogen, carbon monoxide, nitric oxide and cyanogen, are the only ones in which this double kernel within a single octet is possible. That this is so is also suggested by the symmetrical way in which carbon monoxide, nitric oxide, and cyanogen are related to nitrogen, the element which lies just between $C$ and $O$ in the periodic table. With the exception of these four substances having a special structure and such an obviously unsaturated compound as nitrogen dioxide we have found that all the ordinary compounds of carbon, hydrogen, nitrogen and oxygen fit in well with the simple octet theory. This theory accomplished exactly what we stated the ideal theory of atomic structure should do-namely, it leads automatically to a valence theory for carbon-hydrogen-oxygen compounds which is identical with that in common use, while at the same time it indicates that this ordinary valence theory is inapplicable to nitrogen-oxygen compounds. The octet theory on the other hand applies equally well to nitrogen-oxygen compounds as to organic compounds. In all cases it leads to more definite conceptions of the structure of molecules and compounds and explains their chemical and so-called physical properties very much more completely than the older valence theory was ever able to do.

Let us now continue with a consideration of the properties of the elements and their compounds.

Fluorine $N=9, E=7 .-$ The fluorine atom has 7 electrons in its shell. Its properties are therefore largely determined by the tendency to take up an additional electron to complete the octet. In the elementary state two atoms combine together to form a molecule because by so doing each atom can complete its octet. Thus if the molecule is $\mathrm{F}_{2}$ we have $n=2 ; e=\mathrm{I} 4$, whence $p=\mathrm{I}$. By sharing a single pair as shown in Fig. 13, both octets are completed.

The very low boiling point of fluorine indicates that the stray field around this molecule is small. On the other hand the remarkable chemical activity shows that there is a strong tendency for these atoms to avoid sharing their electrons with each other. Thus when lithium and fluorine 
are brought together, the extra electrons from the lithium atoms are taken up by the fluorine atoms and thus each atom is able to get its own octet instead of being compelled to share it with another atom. The very large heat of formation of lithium fluoride (about I I0,000 calories per gram molecule) must be, in greater part, the heat equivalent to the difference between a free octet and one which shares two of its electrons. The energy liberated when an atom of fluorine, with its 7 electrons in the shell, takes up another electron would be greater than the above by the energy required to dissociate fluorine into atoms - a quantity which has never been determined but which must be very large.

The question arises, why do atoms share their electrons with each other, if there is such a tendency for the octets to stay separate. The answer, of course, is that there are not enough electrons in the outside shells of atoms to form octets around their kernels unless they share them with each other. Furthermore most atoms if they completed their octets, without.sharing electrons would have very high charges on their atoms which would tend to prevent their formation.

Hydrofluoric Acid, HF.-The electrons from the hydrogen allow the fluorine atoms to have separate octets. The hydrogen nucleus is then held by a pair of the electrons forming the octet. The large heat of formation of hydrogen fluoride (38,000 calories) notwithstanding the fact that hydrogen atoms have to dissociate during the process, indicates that with fluorine there is little tendency to avoid sharing one of the pairs of the octet with a hydrogen nucleus. This is another illustration of the fact brought out in Postulate 9 that the tendency to form a stable pair between a kernel and a hydrogen nucleus (Case $c$ ) is much greater than the tendency to form a pair between two kernels (Case $d$ ).

The molecule of hydrogen fluoride from its structure should have a small stray field. Most of its surface is like that of a neon atom and the part where the hydrogen nucleus is resembles that of a hydrogen molecule. However, the lack of symmetry should increase the stray field much above that of either neon or hydrogen. We should thus expect HF to be a gas or liquid not greatly different from water in its boiling point. As a liquid it should be a non-conductor, but because of its polar character, it forms an electrolyte when dissolved in water. That it forms a rather weak acid in water solution is, I think, due to the small volume of the fluorine atom which greatly increases the electric force tending to prevent dissociation. The whole question of the effect of atomic volumes on the properties of the atoms will be discussed in another place.

Lithium Fluoride, $\mathrm{LiF}$.-This consists of positively charged lithium kernels and negatively charged fluorine atoms. It is therefore a solid salt, which conducts electrolytically when molten.

Carbon Tetrafluoride, $\mathrm{CF}_{4}--\mathrm{By}$ applying the octet theory taking $n=5$, 
$e=32$, and we find $p=4$. Or we can take $n=4$ and get $p=0$. In the first case the carbon atom shares its electrons with 4 fluorine atoms, while in the second it gives up 4 electrons to the fluorine atoms, these fluorine ions being then held by electrostatic forces to the positively charged carbon kernel. In general the first structure would be much more probable but in view of the exceptional properties of fluorine and the difficulty with which it shares electrons it may well be that the second structure applies to this case.

Nitrogen or oxygen alone does not form any compounds with fluorine: Together they form $\mathrm{NO}_{2} \mathrm{~F}$ and $\mathrm{NOF}$, both gases resembling fluorine in their chemical activity. This fact is very interesting, for $\mathrm{NO}_{2}$ and $\mathrm{NO}$ are remarkable in that they both exist separately but have an odd number of electrons. The fluorine atom, which has an abnormally large affinity for an electron, takes the odd electron from these substances even though it is incapable of combining with oxygen or nitrogen in any other form.

The reasons that fluorine does not form compounds with nitrogen, oxygen or chlorine seem to be as follows:

Helium and neon have the most stable atoms of any of the elements. More energy must be expended to remove an electron from these atoms than from any of the others. The elements hydrogen and fluorine which differ from helium and neon in having one electron less should be expected to have the strongest tendencies to take up electrons. However, this argument needs a radical modification in the case of the hydrogen atom for the charge on this nucleus is only half that on the helium nucleus. The tendency for the hydrogen atom to take up an electron will therefore be very much less than that of the helium atom to hold its electrons. In the case of fluorine and neon this effect is not so serious for the charge on the fluorine kernel is $7 / 8$ of that on the neon kernel.

There is another factor, moreover, which largely eliminates the electronegative character of hydrogen, namely, that the stable pair of electrons held by the hydrogen nucleus can at the same time form part of an octet. For example, consider what must happen if we bring together a hydrogen atom and a fluorine atom. Let us suppose for a moment that the hydrogen atom has such a great affinity for an electron that it takes an electron from the fluorine, leaving this with only 6 electrons. The positively charged fluorine then attracts the negatively charged hydrogen, and, since the stable pair can at the same time form part of an octet, the fluorine is then able to bind the hydrogen nucleus together with its pair. For this reason the tendency of the hydrogen nucleus to take up electrons does not interfere with other electronegative elements getting theirs. Hydrogen therefore can hardly be classed as an electronegative element.

According to our theory therefore fuorine should be the most strongly 
electronegative element, while for similar reasons chlorine and oxygen should come next.

Fluorine in the free state, $F_{2}$, already has enough electrons to form an octet about each atom. The atoms however strive to get a structure exactly like that of neon, that is, one in which each atom has 8 electrons of its own. This means that fluorine has comparatively little tendency to combine with other elements except insofar as it can acquire its electrons outright. That is, it has a strong tendency to take electrons from other atoms instead of sharing them with other atoms. Now oxygen, nitrogen, and chlorine, as elements as well as in their compounds, always have octets around their kernels. The fluorine molecule therefore in order to take electrons from these electronegative elements must break down these stable octets. Undoubtedly atomic fluorine would be able to do so, but it is natural enough that molecular fluorine which already has an octet for each atom (although shared) should not be able to accomplish this. On the other hand elements like boron, silicon, phosphorus, and sulfur form some compounds in which their atoms do not contain octets, but hold the surrounding atoms by means of their charge. The charge on the kernels of these elements is not so great but that fluorine can take their electrons. It is true that the fluorine compounds of carbon and silicon and a few compounds with other elements do seem to involve a sharing of electrons between the fluorine atom and that of the other element, but it is probable that it is easier to share electrons with atoms having small charges on their kernels than with those having large charges.

The fact that fluorine prefers to share electrons with its own atoms rather than with those of any except more electropositive elements such as carbon, phosphorus, etc., is probably due mainly to the greater symmetry of the molecule formed in this way.

Neon.-The atoms of this element already having complete and separate octets have no tendency to take up or give up electrons nor to share them with other atoms. Neon thus forms no primary valence compounds. However there is a stray field of force around the atoms and this accounts for the liquefaction and solidification of the gas at low temperatures.

\section{Second Short Period.}

The atoms of the elements beyond neon easily give up electrons and revert to the form of neon. The first two elements, sodium and magnesium, have properties which are practically wholly dependent on this giving up of electrons. With increasing numbers of electrons the larger electrostatic forces make it more and more difficult for the atom to revert to that of neon, but tend rather to make the atom take up additional electrons to form a structure like that of argon. According to ordinary potential theory, electrons uniformly distributed throughout a spherical 
shell should exert no forces on electrons inside the shell, but should repel those outside the shell as though the electrons in the shell were concentrated at the center. On the other hand, an electron in the spherical shell itself is repelled by the others in the shell as if one-half of the other electrons were removed altogether, while the second half were concentrated at the center. Thus let us consider a carbon atom $(N=6)$ which has taken up 4 extra electrons and has completed its octet. An electron in the outside shell is thus attracted by the nucleus which has 6 positive charges, is repelled by the two electrons in the first shell as though they were concentrated at the center, and is repelled by the 8 electrons in the outside shell as if 4 of them were concentrated at the center. The repulsion of the electrons is thus only just able to neutralize the attraction by the nucleus, notwithstanding the fact that the whole atom has an excess of 4 negative charges. If, instead of taking carbon, we had considered nitrogen there would have been a large resulting force tending to hold the electrons even after 3 extra ones had been taken up by the atom. These considerations indicate one of the reasons why the symmetrical arrangements of electrons corresponding to the inert gases have such very great stability and why the elements like oxygen and especially fluorine have such very great tendencies to take up electrons.

According to the above calculation, carbon might just be able to hold 4 electrons, while boron could not do so. In the crude theory just given, however, we have neglected to take into account the formation of pairs when electrons are shared between octets or between these and hydrogen nuclei. This will greatly increase the tendency of an atom like that of carbon to take up electrons to complete its octet. For this reason we also find a few boron compounds such as borofluoric acid $\left(\mathrm{HBF}_{4}\right)$, metaboric acid, etc., in which the boton atom has an octet. These same arguments apply to such elements as aluminum and silicon.

Atomic Volumes.-The properties of the elements lithium, beryllium, and boron are not as closely related to those of sodium, magnesium and aluminum as these are to potassium, calcium and scandium. Instead we find very marked resemblances between lithium and magnesium, beryllium and aluminum, and boron and silicon. These differences and similarities ate due, I think, to the small atomic volumes of lithium, beryllium and boron in their compounds. Before considering these elements individually a discussion of atomic volumes will not be out of place.

The periodic relationships between the atomic volumes of the elements are usually based on the volumes of the free elements in solid or liquid form. ${ }^{1}$ Because of the radically different structures which occur among these substances these measurements give very little information in

1 Tables and curves giving these atomic volumes have been given by Harkins \& Hall, This Jourana, 38, ig6 (1916). 
regard to the real volume of the atoms. Thus the structure of fluorine with its diatomic molecules is so different from that of metallic sodium that effects due to the difference in constitution may mask any real changes in the volume of the atoms. This difficulty is largely avoided if we consider the volumes of the atoms in the form of similarly constituted compounds.

In view of the very great stability of the octet in neon it seems probable that the volume of this octet is not materially different from the complete octets in the fluorine and sodium ions. The closeness to which two octets can be made to approach each other however should depend on how great the forces are which draw them together. In liquid neon these forces are weak, so the atoms should be at comparatively great distances. The atomic volume (i.e., atomic weight divided by the density of the liquid) of neon is I9.2. Sodium fluoride consists of sodium and fluorine ions held together in a space lattice by the positive and negative charges. Both ions contain complete octets which differ from those of neon only because of the slightly different charges on the kernels. It may be assumed that the fluorine and sodium octets contribute equally to the molecular volume of sodium fluoride which is 15.2. Thus while in liquid neon the volume is 19.2 in sodium fluoride the volume occupied by each octet is 7.6 . It is probable that the volume of the octet itself, that is, the volume of the cube formed by the electrons, is not materially different in the two cases but the strong forces in the sodium fluoride draw the octets closer together. Magnesium oxide whose molecular volume is II.O, consists of oxygen atoms with a double negative charge and magnesium atoms which have lost two electrons-in both cases giving a simple octet with a structure like that of neon. The volume occupied by each octet is thus 5.5 as compared to 7.6 in sodium fluoride and 19.2 in liquid neon. These results give a measure of the extent to which octets may be made to approach each other more closely by strong forces. Comparing magnesium oxide and sodium fluoride the relative distances between the atoms are as the cube roots of their volumes or as I is to I.II4. The ratio of the forces acting between the atoms, taking account of the double charges of the magnesium and oxygen atoms, is as one is to 4.98 . Thus for a 5 -fold increase in the force, the volume occupied by the octets has decreased $27 \%$ ( 7.6 to 5.5 ). The atomic volume of metallic sodium is 23.7 , greater than even that of neon, notwithstanding the fact that the charges on the electrons and the positively charged sodium ions might be expected to exert strong attractive forces compared to those in liquid neon. It is not reasonable to suppose that the actual volume of the octet of the sodium atoms in metallic sodium is much different from that in sodium fluoride. The large volume of metallic sodium must then be due to a specific repulsion between a completed octet and a single free electron which keeps 
the electron from approaching the octet. It is probable that this same repulsion is the cause of the remarkable and apparently perfect elasticity of the collisions between electrons and the atoms of the inert gases, or nitrogen. ${ }^{1}$ The fact that nitrogen is similar to the inert gases in this respect is additional confirmation of our theory that the nitrogen molectle has a single octet.

It is especially interesting to compare the volume of sodium fluoride with that of metallic sodium for in each case the substance is held together by the forces between particles having unit charges. The volume occupied by the sodium ion in sodium fluoride is 7.6 . The free electron in metallic sodium increases this to 23.7 so that the electron seems to require a volume of I6.I, or more than twice that of the octet. However, the effect is probably not quite as great as this for the distance between the positive and negative charges in sodium is some $15 \%$ greater than in sodium fluoride. The actual space lattice arrangement of the atoms also needs to be known and taken into account in any proper treatment of this subject. From the molecular volume of magnesium oxide it appears that the atomic volume of the octet is less than 5.5 -it is reasonable to assume that with much larger forces it might approach a limiting value of about 4.0. The volume of a single neon octet is thus $6.2 \times 10^{-24} \mathrm{cc}$. corresponding to a cube having an edge of $1.9 \times 10^{-8} \mathrm{~cm}$.

Assuming the electrons and the sodium ions in metallic sodium to be arranged like the ions of chlorine and sodium in a crystal of sodium chloride, we find that the distance between their centers must be $2.7 \times$ $10^{-8} \mathrm{~cm}$. Presumably at distances shorter than this the repulsive forces between a single electron and an octet more than compensate for the attraction between oppositely charged univalent ions.

According to this view the large atomic volumes of the alkali metals are due to the volumes occupied by single free electrons rather than to a large volume of the atom as such.

Magnesium, although there are two free electrons for each atom, has an atomic volume of 14.0, only a little more than half that of sodium. Comparing this with magnesium oxide, of molecular volume $I$, it is clear that the electrons contribute much more to the volume of the metal than does the octet of the oxygen in the oxide. It is probable that the two free electrons form a more or less stable pair and are thus able to occupy a smaller volume than the single electron in sodium.

The atomic volumes of the elements increase at first about in proportion to the atomic numbers and then more slowly. For example, the atomic volumes of the free alkali metals are: lithium I3.1, sodium 23.7, potassium 45.5 , rubidium 56.0 , and cesium 69.8 . The volumes in their compounds are seen from: sodium fluoride ${ }_{15.2}$, potassium chloride 37.4 ,

'Franck and Hertz, Verh, deut. physik. Ges., 15, 929 (1913). 
rubidium bromide 5 1.4, and cesium iodide 57.5 . In these compounds the two elements are chosen so as to have as nearly the same atomic numbers as possible. Half of these quantities should give a measure of the maximum atomic volumes of the inert gases lying between them. In this way we find neon 7.6, argon 18.7 , krypton 25.7 , and xenon 28.7. The molecular volume of lithium fluoride is only Io.o, while that of the fluorine atom alone in sodium fluoride is apparently 7.6. The atomic volume of the lithium nucleus thus must be very small, probably about 2.4. The same rapid increase in atomic volume between the first and second period is observed with the elements in the middle of the period, thus boron 4.7 , aluminum 10.4, and carbon 3.4, silicon II.7.

It seems as though the total volume of the atom tends at first to increase in proportion to the number of electrons. This means that the cells of Postulate 3 have the same volumes even in different atoms in spite of the increasing attraction of the nucleus. However, with larger atomic numbers the rate of increase of volume is much less, and with certain elements like osmium notwithstanding the high atomic number, the atomic volume is so small (8.5) that the hypothesis of constant cell volume seems untenable. It should be noted that the elements of small atomic volume are those in which there are larger numbers of electrons in the outer shell held by attractive forces (Postulate 6) to the underlying electrons. When there are either too few electrons, or when the electrostatic forces predominate, the atomic volume increases.

Let us now continue with a discussion of the properties of individual elements.

Sodium $N=$ I I, $E=$ I. - The properties are determined almost solely by the ability of its atoms to give up one electron. It is much more electro-positive than lithium because its atomic volume is greater so that the force with which it holds its electron is smaller.

Magnesium $N=12, E=2$. - The properties are largely dependent on the ability to give up two electrons. The double charge makes this process more difficult than with sodium. There is thus a greater tendency to form insoluble salts and for hydrolysis to occur. Lithium, because of its small volume, holds its electrons more firmly than does sodium, and for this reason resembles magnesium in the solubility of its salts, etc. Magnesium has no tendency to form an octet.

Aluminum $N=13, E=3$. - The difficulty of giving up 3 electrons to form a positive ion leads to marked hydrolysis and to a tendency to form insoluble salts. In compounds with oxygen, aluminum probably forms an octet as, for example in $\mathrm{KAlO}_{2}$. If we place $n=3, e=\mathrm{I} 6$, we find $p=4$ and are led to the formula $\mathrm{K}^{+}(\mathrm{O}=\mathrm{Al}=\mathrm{O})^{-}$. $\quad$ Beryllium, with only two electrons in its outside shell, has a kernel of such small volume that it holds its electrons nearly as firmly as aluminum and 
therefore resembles aluminum in the solubility of its salts and in other ways.

Silicon $N={ }_{14}, E=4$.- - This element is no longer able to form positive ions but like carbon, forms practically only compounds in which its atoms have octets. Boron, owing to its small volume, holds its electrons about as firmly as silicon and therefore resembles this element.

The chemistry of silicon compounds is complicated by the marked tendency to form second order compounds. The great number of silicates found in nature seem to be compounds of silicon dioxide with other oxides. The octet theory is applicable to the formation of silicon dioxide and each of the other oxides but not to the minerals resulting from their combination. By far the greater number of such compounds exist in the solid state only. The number of molecules of each of the oxides that can combine together in this way to form crystalline solids is determined mainly by purely geometrical considerations. The writer has already discussed compounds of this kind at some length ${ }^{1}$ and Sosman ${ }^{2}$ has developed a similar theory.

A compound like hydrofluosilicic acid differs from those we have just considered in that it exists in aqueous solutions. The formation of such compounds is explained by Werner's theory of supplementary valence, but without leading to a definite conception of the mechanism of the combination. We have seen that fluorine has a particularly strong tendency to take electrons outright rather than to share them with other atoms. Thus when fluorine reacts with silicon, 4 fluorine atoms take the 4 electrons from a single silicon atom. The fluorine ions are then held by electrostatic forces and surround the positively charged silicon kernel probably in a tetrahedral arrangement, but without sharing electrons. The electric field is thus almost entirely enclosed within the molecule so the substance $\mathrm{SiF}_{4}$ is a gas of rather low boiling point. The silicon kernel having as its second shell an octet like that in neon, has a cubic symmetry. There is thus a tendency for the kernel to take up 6 rather than 4 fluorine ions since these ions can fit opposite the 6 faces of the cube in a very symmetrical way. It may at first seem that a quadrivalent positive ion could not hold 6 negative ions by electrostatic forces but close examination indicates that this arrangement should be possible. Let us assume that a silicon kernel has taken up 6 fluorine ions to form the complex ion $\mathrm{SiF}_{6} \cdots \cdots$. What are the forces acting on each fluorine atom? Let us imagine the fluorine ions at the 6 corners of a regular octahedron with the silicon ion at its center. We will take the distance from the center to the corner of the octahedron as the unit of length. Let us consider the forces acting on one of the fluorine ions which we will denote

1 This Journal, 38, 2241 (1916).

"J.Ind. Eng. Chem, 8, 985 (1916). 
by $A$. The attractive force between $A$ and the quadrivalènt silicon ion is 4. The repulsive force between $A$ and the fluorine ion farthest from $A$ is $1 / 4$ since the distance is 2 . Each of the other 4 fluorine ions is at a distance $\sqrt{2}$ from A so that the force is $1 / 2$. But the component in the direction towards the center is only $\mathrm{I} /(2 \sqrt{2})$. The 4 fluorine ions thus exert a total repulsive force of $2 \sqrt{2}$ or I.4I. All 5 thus repel the ion A with a force $1.4 \mathrm{I}+0.25=\mathrm{I} .66$, while the silicon atom attracts it with a force of 4. Thus notwithstanding the negative charge on the complex ion as a whole, each fluorine ion is attracted to the central nucleus as strongly as it would be to a simple positive kernel having a charge of 2.34 units. The $\mathrm{SiF}_{6}-{ }^{-}$ion can exist as such in solution or can attach to itself two hydrogen ions to form $\mathrm{H}_{2} \mathrm{SiF}_{6}$.

This simple theory indicates how compounds with a coördination number of 6 can be formed because of purely geometrical and electrical factors. The forces causing such combinations as well as those holding complex silicates, etc., together, will be referred to as secondary valence forces.

The marked contrast between silicon and carbon in their tendencies to form second order compounds is probably due to the larger volume of the kernel of the silicon atom. This probably greatly decreases the tendency of the silicon atom to hold an octet when combined with oxygen as silicon dioxide. We may picture a molecule of silicon dioxide diagrammatically as in Fig. I4. When a second octet forms around the silicon kernel it would normally have a larger spacing between its electrons than those usual in an oxygen atom. When the silicon and oxygen atoms hold two pairs of electrons in common the electrons of the silicon atom are drawn over towards the oxygen. This exposes the positively charged silicon kernel so that a large external field results. This effect is like

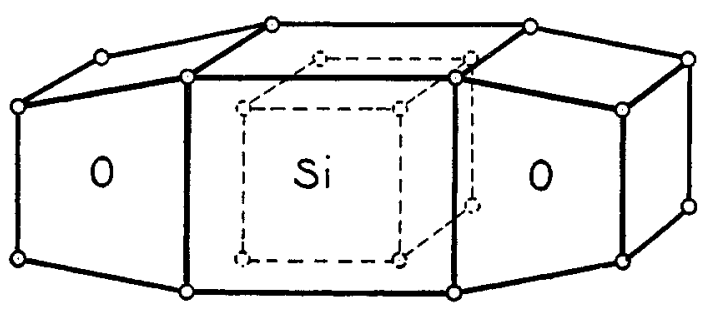

Fig. 14.

Diagram of silicon dioxide. that caused by the double and especially the triple bonds in organic compounds. There is a continuous series of graduations between a structure of this kind and one in which two oxygen atoms, each with a double negative charge, are held electrostatically by the positively charged silicon kernel. In either case however the oxygen atoms, because of their small number and small volume, are not able to surround the silicon kernel and make its field nearly all internal as is the case in the carbon dioxide molecule. The large external field thus causes molecules of silicon 
dioxide to be held very firmly to each other or to any other similarly constituted oxides. On the other hand, when oxygen atoms completely surround a kernel of small volume as, for example, in osmium tetroxide, the weak external field causes the substance to have a low boiling point (about $100^{\circ}$ for $\mathrm{OsO}_{4}$ ). When the silicon atoms combine with the halogens or with hydrogen the larger number of these atoms allows them more nearly to surround the silicon atom and this leads to the formation of liquid and gaseous products, in which secondary valence forces are much less manifest.

Phosphorus $N={ }_{15}, E=5$.- The phosphorus atom like that of nitrogen has 5 electrons in its shell. The peculiar arrangement by which two nitrogen atoms form a molecule with a single octet containing an imprisoned pair of electrons is impossible in the case of phosphorus because of the complexity and large volumes of the kernels. Phosphorus therefore cannot form molecules of the composition $\mathrm{P}_{2}$ except by the structure $\mathrm{P} \equiv \mathrm{P}$. It also cannot form $\mathrm{P}_{3}$ for this involves an odd number of electrons. Let us examine by the octet theory the possibility of forming $\mathrm{P}_{4}$. Here $n=4, e=20$ and $p=6$. The only reasonable

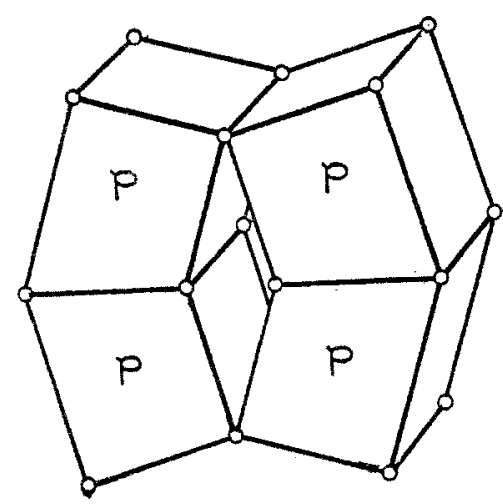

Fig. 15 .

Diagram of the phosphorus molecule $P_{4}$ ways in which 4 atoms can be arranged sharing 6 pairs are represented by $P=$ $\mathrm{P}=\mathrm{P}=\mathrm{P}$ and $\mathrm{P}-\mathrm{P}$

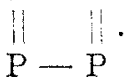

Phosphorus vapor actually has the composition $\mathrm{P}_{4}$. It is probable from reasons of symmetry that its molecule is represented by the second of the above formulas as indicated in Fig. 15.

Let us now apply the octet theory to the various typical compounds of phosphorus. It will be seen that in every case results are obtained in full agreement with the properties of the compounds without any special assumptions

in regard to valence. The structural formulas obtained are in most cases quite different from those derived from the ordinary theory.

Phosphorus Hydrides, $\mathrm{P}_{4} \mathrm{H}_{2}, n=4, e=22, p=5$. This gives by analogy with $\mathrm{P}_{4}$ the formula $\mathrm{P}=\mathrm{P}$

$$
\mathrm{HP}-\mathrm{PH}
$$

For phosphine, $\mathrm{PH}_{3}, n=1, e=8, p=0$; hence, $\mathrm{PH}_{3}$.

For liquid hydrogen phosphide $\mathrm{P}_{2} \mathrm{H}_{4}, n=2, e=14, p=1$; hence, $\mathrm{H}_{2} \mathrm{P}-\mathrm{PH}_{2}$. 
Phosphorus Oxides.-The suboxide $\mathrm{P}_{4} \mathrm{O}$ gives $n=5, e=26$ and $p=7$, for which by analogy with $\mathrm{P}_{4}$ we find the structural formula to be $\begin{array}{lll}\mathrm{P}=\mathrm{P} & \text { or } & \mathrm{P}=\mathrm{P} \\ \mathrm{P}=\mathrm{P}-\mathrm{O} & \mathrm{P} & \mathrm{P}-\mathrm{P}=\mathrm{O}\end{array}$

The trioxide has a vapor density corresponding to $\mathrm{P}_{4} \mathrm{O}_{6}$. This gives $n=10, e=56$ and $p=12$. Since this oxide is obtained by the partial oxidation of $\mathrm{P}_{4}$ and from reasons of symmetry it is probable that its constitution is<smiles>O=[P-]1P(=O)(O)P(O)[PH]1(O)O</smiles>

In this formula there are 12 pairs of electrons held in common, in accordance with $p=12$. Each phosphorus atom not only has its octet, but has all 4 pairs of electrons in each of these octets shared by other atoms. We shall see that in practically all phosphorus compounds just as in carbon compounds there is a strong tendency for the 4 pairs in the octet of the central atom to be shared. In fluorine we have noticed just the opposite tendency, namely, not to share any of the pairs with other atoms.

If we represent a molecule of the trioxide by $\mathrm{P}_{2} \mathrm{O}_{3}$ its constitution can be written $\mathrm{O}=\mathrm{P}-\mathrm{O}-\mathrm{P}=\mathrm{O}$. This should be rather unsaturated as compared to the ring since only 3 pairs of electrons around each phosphorus atom are shared. This formula, however, shows the relationship of the trioxide to the acids of phosphorus better does than the ring formula. A reasonable transition between the two structures is $\mathrm{O}-\mathrm{P}-\mathrm{O}-\mathrm{P}-\mathrm{O}$<smiles>C=P(O)(P)OPP(O)O</smiles>

Phosphorus Pentoxide.-The vapor density indicates the formula $\mathrm{P}_{4} \mathrm{O}_{10}$. This gives $n=14, e=80$ and $p=16$. From its derivation

from $\mathrm{P}_{4} \mathrm{O}_{6}$, the most probable constitution is<smiles>O=[P]1O[P](O)(O)O[P](O)(O)O[P@]1(=O)O</smiles>

If the formula of the pentoxide is taken as $\mathrm{P}_{2} \mathrm{O}_{6}$ we can write its formula $\mathrm{O}$ $\mathrm{O}$ $\mathrm{O}=\mathrm{P}-\mathrm{O}-\mathrm{P}=\mathrm{O}$. It is readily seen that the octet theory indicates that no oxides higher than the pentoxide should exist. 
Acids of Phosphorus.-The following table shows the constitution of the various acids as given by the octet theory.

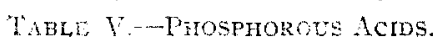

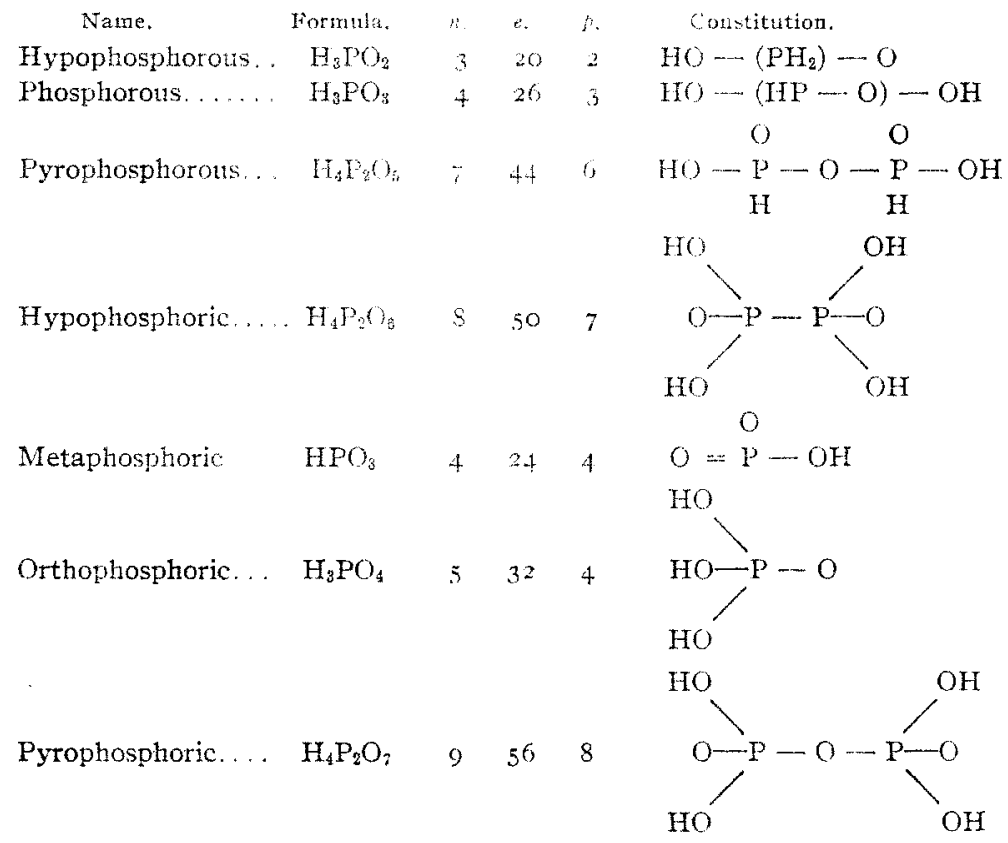

The constitution as determined from the values of $p$ is shown more clearly in Fig. 16. In each case all 4 pairs of the phosphorus octets are shared by the adjacent atoms. It is significant that these formulas give correctly the number of replaceable hydrogen atoms. Thus hypophosphorous acid is a monobasic acid, while phosphorous and pyrophosphorous acids are dibasic. This is readily explainable by the formulas, for it is seen that in the first case two of the hydrogen atoms and in the other cases one atom is bound directly by each phosphorus atom and therefore does not show acid properties.

Phosphorus Chlorides.-For the trichloride $\mathrm{PCl}_{3}$, we find $n=4$, $e=26$ and $p=3$. The phosphorus octet thus shares a pair of electrons with each of the 3 chlorine atoms. Here is one of the few cases in which the phosphorus atom does not share 4 pairs. It is readily seen from the octet theory, however, that phosphorus could not form a compound $\mathrm{PCl}_{4}$. Since the chlorine atoms can nearly surround the phosphorus atom and each atom has its octet, the trichloride molecule has a weak external field and hence has a fairly low boiling point $\left(76^{\circ}\right)$.

For phosphorus pentachloride $\mathrm{PCl}_{5}$, we place $n=5, e=40, p=0$. This leaves the phosphorus kernel without an octet, the 5 chlorine ions being held by electrostatic attraction. The external field is weak and the 
substance evaporates easily. The tendency of the phosphorus kernel to acquire an octet even if has to take two electrons from chlorine ions makes this compound dissociate easily into the trichloride and chlorine. If instead of placing $n=5$ we place $n=6$, then $e=40$, and we find $p=4$. The 5 chlorine atoms cannot share 4 pairs of electrons with the phosphorus octet, but we can imagine that in the solid state of phosphorus pentachloride 4 of the chlorine ions share electrons with the
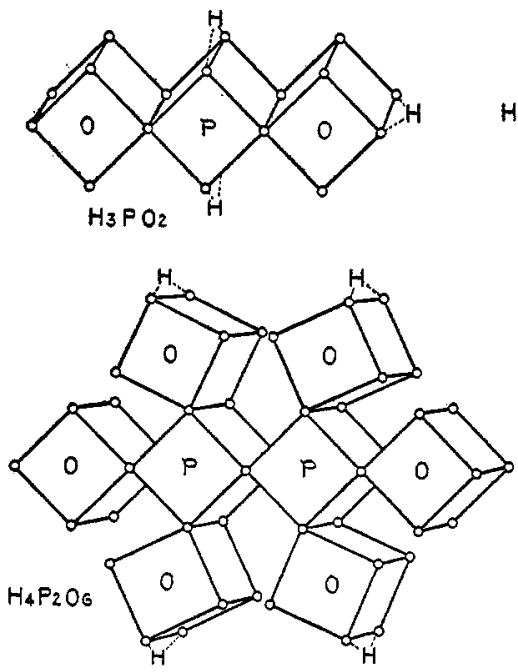
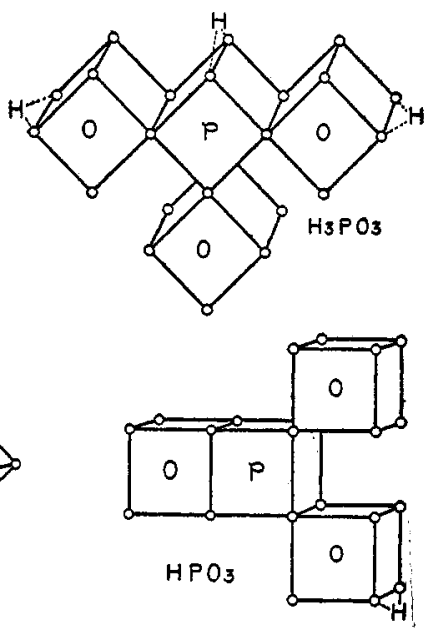

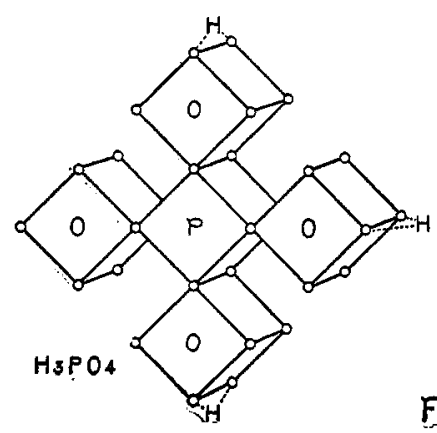

Fiq. 16.

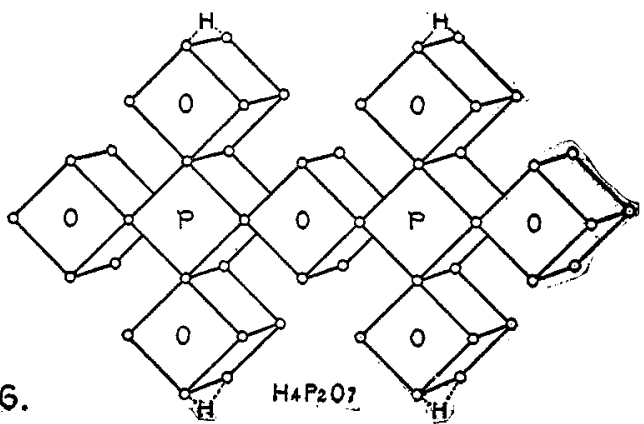

$\mathrm{H}_{4} \mathrm{P}_{2} \mathrm{O} 3$

Diagrams of molecules of phosphorous oxy-acids.

phosphorus octet to form an ion $\left(\mathrm{PCl}_{4}\right)^{+}$, while the other chlorine ion is held in the space lattice by electrostatic forces. It is perhaps probable that the structure of solid phosphorus pentachloride is of this kind. If so, the high melting point $\left(148^{\circ}\right)$ of phosphorus pentachloride compared with the trichloride $\left(-\mathrm{II}_{1}{ }^{\circ}\right)$ is explained by the large electrostatic forces holding the $\mathrm{PCl}_{4}{ }^{+}$and $\mathrm{Cl}^{-}$ions to each other and by the symmetry of the $\mathrm{PCl}_{4}+$ ion. However, liquid phosphorus pentachloride is a non-con- 
ductor of electricity so that in this state only molecules of the type $\mathrm{PCl}_{5}$ exist.

In the compound $\mathrm{POCl}_{3}$ we find that all the atoms can complete their octets without difficulty. Thus $n=5, e=32, p=4$ so that each of the 4 atoms shares one pair of electrons with the octet of the phosphorus atom. This is the reason that phosphorus tends so strongly to form this type of compound with the halogens.

Sulfur $N=I 6, E=6$.- Since the shell contains 6 electrons like that of oxygen, we might expect sulfur to form a molecule, $S_{2}$. At very high temperatures, sulfur vapor has a density corresponding to this formula, but at temperatures a little above the boiling point the vapor consists mostly of $\mathrm{S}_{3}$, which is also the molecule found from cryoscopic measurements. For this molecule the octet theory gives $n=8, e=48$, and $p=8$. By forming a ring compound the 8 electrons can be held together by single pairs of electrons, whereas if we arrange the 8 atoms in a chain there must be one double bond. The ring structure being much more symmetrical is more probable. The most symmetrical formulas seem to be:- $-I$, a continuous ring of 8 electrons arranged in space as shown in Fig. I 7 . This structure allows the secondary as well as primary valence forces to be satisfied. The molecule probably draws itself together rather more compactly than shown in the figure and thus forms a very symmetrical structure resembling a regular tetrahedron; II, the second possible ring formula is

$$
s-s-s-s
$$$$
s-s-s-s
$$

Although this has a superficial resemblance to the structures best representing the $\mathrm{P}_{4}$ molecule, it seems unlikely that it corresponds to the constitution of $\mathrm{S}_{8}$. We shall see that the tendency of the stulfur atoms to form chains is a characteristic property of this element.

Polysulfides.--The tendency by which oxygen atoms attach themselves to each other forming compounds like ozone and hydrogen peroxide, is exhibited in still greater degree by sulfur atoms. Thus there is a series of sulfides of sodium represented by $\mathrm{Na}_{2} \mathrm{~S}_{x}$ where $x$ may have values up to 5. Placing $n=5, e=32$, and we find $p=4$. The most probable structure is

$$
\mathrm{Na}+\left[\begin{array}{c}
\mathrm{S} \\
\mathrm{s}-\mathrm{S}-\mathrm{s} \\
\dot{S}
\end{array}\right]^{--} \mathrm{Na}^{+}
$$

The octet theory thus explains the formation of the sulfides from $\mathrm{Na}_{2} \mathrm{~S}$ to $\mathrm{Na}_{2} \mathrm{~S}_{5}$ and indicates that more than 5 sulfur atoms in the anion would require a more complicated structure. 
Oxides of Sulfur.-For sulfur dioxide we find $n=3, e=18, p=3$, giving the structure $\mathrm{O}=\mathrm{S}-\mathrm{O}$. For the trioxide, $\mathrm{SO}_{3}$, we have $n=4$, $e=24, p=4$, and thus find $\mathrm{O}=\mathrm{S} \mathrm{O}_{\mathrm{O}}^{\mathrm{O}}$. This is the most stable oxide because all 4 pairs of electrons of the sulfur octet are shared by the oxygen atoms. It is readily seen from the octet theory that the molecules $\mathrm{O}=\mathrm{S}\left\langle\succ_{\mathrm{O}}^{\mathrm{O}}\right.$ should show a tendency to polymerize to form long chains having the structure

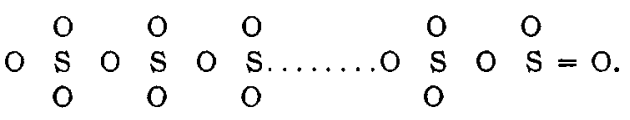

The more stable solid modification of sulfur trioxide, which consists of long, fibrous crystals probably has this structure.

The very unstable sesquioxide $\mathrm{S}_{2} \mathrm{O}_{3}$, which is formed as a blue liquid or bluish green solid, when sulfur is dissolved in liquid sulfur trioxide, at I $2^{\circ}$, gives by the octet theory $n=5, e=30, p=5$. It thus probably has the composition $\mathrm{S}-\mathrm{O}=\mathrm{S}>_{\mathrm{O}}^{\mathrm{O}}$ and may thus be regarded as a persulfide of sulfur trioxide. The blue color, which indicates an unstable arrangement of electrons, is probably quite analogous to that produced by the action of hydrogen peroxide on chromates.

Persulfuric anhydride, $\mathrm{S}_{2} \mathrm{O}_{7}$, is a very volatile liquid which solidifies to a mass of long needles at $0^{\circ}$ and decomposes readily into oxygen and sulfur trioxide when heated. The octet theory gives $n=9, e=54$, and $p=9$. From this value of $p$ and from the fact that the product is formed by an electric discharge under conditions which yield ozone, the constitution probably is<smiles>OO[As](O)OS(O)(O)O</smiles>

Oxy-acids. - The constitutions of the ions of these acids as found by the octet theory are given in Table VI. The ions rather than the free acids are tabulated because many of the acids do not exist in the free state. In determining the value of $e$ the charge on the ion must be taken into account.

In the more stable acids all 4 pairs of electrons in the octets of the central atoms are shared by the adjoining atoms. 
TABLE VI.

Ions of the Sulfur Acids.

Name of acid.

Foranนา.

n. $e$.

p. Constitution.

Sulfurous

$\mathrm{SO}_{3}^{--}$

4. 26

3<smiles>O=S(O)O</smiles>

Sulfurio

$\mathrm{SO}_{4}-$

5<smiles>[2H][Si](O)(O)O</smiles>

Hyposulfurous. ........ $\mathrm{S}_{2} \mathrm{O}_{4}^{-\cdots}$

$6 \quad 38$<smiles>OB(O)[SH](O)O</smiles>

Thiosulfuric

$\mathrm{S}_{2} \mathrm{O}_{3} \cdots$

$5 \quad 32$<smiles>O[As](O)(O)S</smiles>

Pyrosulfuric

$\mathrm{S}_{2} \mathrm{O}_{7}^{-}$

$9 \quad 56$<smiles>OS(O)(O)OS(O)(O)O</smiles>

Dithionic. ....... $\mathrm{S}_{2} \mathrm{O}_{6}-\cdots \quad 8 \quad 50$<smiles>OS(O)(O)S(O)(O)O</smiles>

Trithionic...... $\mathrm{S}_{8} \mathrm{O}_{6}-{ }^{-} \quad 96$<smiles>OS(O)(O)SS(O)(O)O</smiles>

Tetrathionic. ..... $S_{4} \mathrm{O}_{5} \cdots \quad 10 \quad 62$<smiles>OS(O)(O)SSS(O)(O)O</smiles>

Pentathionic...... $\mathrm{S}_{5} \mathrm{O}_{6} \ldots$ ir 68 ro<smiles>O=[SH](O)(O)SSSS(O)(O)O</smiles>

Persulfuric....... $\mathrm{S}_{2} \mathrm{O}_{8}-\ldots$ io $62 \quad 9 \quad \mathrm{O}-\left.\right|_{\mathrm{S}} ^{\mathrm{S}}-\mathrm{O}-\mathrm{S}-\mathrm{O}$

Halogen Compounds.--The most stable chloride of sulfur is $\mathrm{S}_{2} \mathrm{Cl}_{2}$, for which $n=4, e=26, p=3$. The constitution is thus $\mathrm{Cl}-\mathrm{S}-\mathrm{S}-\mathrm{Cl}$. 
The very unstable chloride $\mathrm{SCl}_{2}$ has the structure $\mathrm{Cl}-\mathrm{S}-\mathrm{Cl}$. The tetrachloride exists as a solid at very low temperatures and has been said to exist as a liquid at $-20^{\circ}$, but dissociates rapidly with rise of temperature into chlorine and $\mathrm{S}_{2} \mathrm{Cl}_{2}$. The octet theory gives for $\mathrm{SCl}_{4}, n=5$, $e=34, p=3$. This indicates that 3 chlorine atoms share pairs of electrons with the sulfur atom and form a positive ion $\left(\mathrm{SCl}_{3}\right)^{+}$while the fourth chlorine atom forms an ion, $\mathrm{Cl}^{-}$. Since the sulfur atom shares only 3 of its pairs of electrons such a compound should be very unstable. There is however very little evidence that it exists, except in the solid state. It is more probable that a very unstable second order compound between $\mathrm{Cl}_{2}$ and $\mathrm{S}_{2} \mathrm{Cl}_{2}$ accounts for the experimental data.

Fluorine has so little tendency to share electrons with other atoms that it forms only one compound with sulfur, namely $\mathrm{SF}_{\theta}$. The symmetrical arrangement of the 6 fluorine ions opposite the 6 faces of the octet of the sulfur kernel, and the fact that the fluorine atom and the sulfur kernel are of about the same size, gives the compound $\mathrm{SF}_{6}$ an extraordinary stability. As a matter of fact, this gas, notwithstanding the large proportion of fluorine in its composition, is a tasteless and odorless gas which is exceedingly stable and inert.

Another tasteless and odorless gas containing fluorine is formed when sulfur dioxide and fluorine are brought into contact with a heated platinum wire. The composition is $\mathrm{SO}_{2} \mathrm{~F}_{2}$. Here $n=5, e=32$, and $p=4$. This gives the constitution $\mathrm{O}-\mathrm{S}-\mathrm{O}$, in which each fluorine atom shares a pair of electrons with the sulfur atom. In this case the tendency of the fluorine to share electrons has been overcome by the combined tendencies of the sulfur atom to take up an octet and to share all 4 of its pairs of electrons with its neighbors.

Chlorine $N=17, E=7$. - The essential differences between chlorine and fluorine seem to be due to the more strongly electronegative character of fluorine and its smaller atomic volume. Chlorine, like fluorine, tends to form negative ions, but it differs from fluorine in that it can share electrons with oxygen, especially if at the same time the molecule takes up electrons from some more strongly electro-positive element. It shows a tendency like phosphorus and sulfur to share all 4 pairs of its electrons, if it has to share any.

Oxides of Chlorine.-The monoxide $\mathrm{Cl}_{2} \mathrm{O}$, according to the octet theory, has the structure $\mathrm{Cl}-\mathrm{O}-\mathrm{Cl}$, since $p=2$. For the heptoxide $\mathrm{Cl}_{2} \mathrm{O}_{7}$, we place $n=9, e=56$, and find $p=8$, giving the structure 


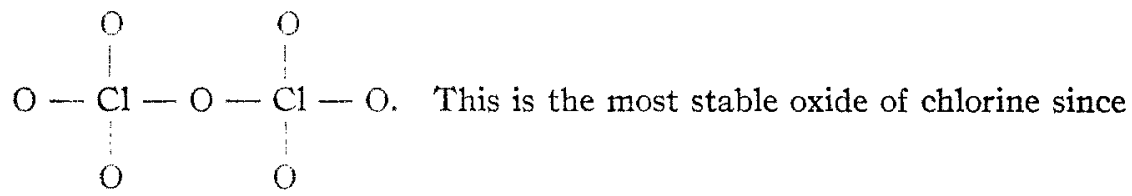

each chlorine shares 4 pairs of electrons. The peroxide $\mathrm{ClO}_{2}$, the least stable of the oxides, contains 19 available electrons. It is thus one of the few compounds that have an odd number of electrons. The only other compounds of this kind we have thus far considered, namely nitric oxide and nitrogen dioxide, had in each case one electron too many to form the normal structures. But chlorine peroxide has one electron too few to form a normal compound of the type $\mathrm{O}-\mathrm{Cl}-\mathrm{O}$. The determination of its structure will be an interesting but probably difficult problem. It is significant that no oxide of chlorine corresponding to $\mathrm{NO}$ is known. This is probably due to the fact that a condensed structure like that of nitric oxide, carbon monoxide, etc, is not possible with atoms having an octet in their kernels.

Oxy-acids. - The octet theory explains immediately the chlorine oxyacids. Thus 4 oxygen atoms can be successively added to the chlorine atom in hydrochloric acid. This gives the acids

$\mathrm{HCl} ; \mathrm{HCl}-\mathrm{O} ; \mathrm{HO}-\mathrm{Cl}-\mathrm{O} ; \mathrm{HO}-\stackrel{\mathrm{Cl}}{\mathrm{C}^{2}}$ and $\mathrm{HO}-\stackrel{\mathrm{Cl}}{\mathrm{C}}-\mathrm{O}$,

0

corresponding exactly to the acids $\mathrm{HN}=\mathrm{O}, \mathrm{HO}-\mathrm{N}=\mathrm{O}, \mathrm{HO}-\mathrm{N}=\mathrm{O}$. In each case the octet theory shows why higher acids cannot be formed. In nitric acid and in perchloric acid all 4 pairs of electrons in the octet of the central atom are shared by the adjacent atoms.

\section{First Long Period.}

Beyond argon we find that the first 3 elements have properties closely related to those of the second short period, but, as was already pointed out in the discussion of the structure of the atoms, these relationships largely disappear beyond titanium. From this point on we find that the octet theory does not apply at all if in calculating $e$ we take the total number of electrons in the shell. For example, all chromic, manganous; ferric and cobaltous salts contain odd numbers of electrons. This diffculty cisappears however if in calculating $e$ in Equation 2 we consider only the available electrons. We have already discussed why only a certain fraction of the electrons should be available in these elements.

There is nothing arbitrary about this choice of the number of available 
electrons. In compounds of iron, chromium, manganese, etc., there are fundamental changes in the character of the compounds whenever the number of available electrons changes. Ferrous and ferric salts, for instance, even in the solid state, are as different from one another as though they were salts of different metals. Their colors, magnetic properties, chemical properties, etc., are all unmistakably different. But among the elements which precede argon there were no such differences. Thus we cannot satisfactorily divide nitrogen, phosphorus or chlorine compounds into classes according to the valence of their parent atom.

In the compounds of vanadium, chromium and manganese in which these elements enter the acid radical, there seems to be a tendency for the central atom to form octets, although the stability of the octet is much less than those formed by phosphorus, sulfur and chlorine. Thus we repeatedly find compounds, $\mathrm{Na}_{3} \mathrm{VO}_{4}, \mathrm{Na}_{2} \mathrm{CrO}_{4}, \mathrm{Na}_{2} \mathrm{MnO}_{4}$ and $\mathrm{NaMnO}_{4}$ in which 4 oxygen atoms surround the central atom. Since the free atoms of these elements have little or no tendency to take up electrons to complete their octets, we must conclude that any kernel with a sufficiently large positive charge ( 5 or more) tends to surround itself with an octet provided all 4 of the pairs of electrons in this octet are shared by adjoining atoms. Thus we may speak of an octet being stabilized by the sharing of its pairs.

Among the elements beyond argon it is common to find that a single element forms several acids corresponding to different numbers of available electrons as illustrated, for example, by $\mathrm{Na}_{2} \mathrm{MnO}_{4}$ and $\mathrm{NaMnO}_{4}$. In the first compound the manganese atom has 6 available electrons while in the second it has 7 . We must picture to ourselves the manganese kernel in the first case as consisting of a simple kernel like that of argon, having 7 positive charges, holding a single electron prisoner within the outer octet that is shared by the 4 oxygen atoms.

It is evident that this sort of thing greatly complicates the application of the octet theory. The difficulty, however, is one that is forced upon us by the actual properties of the elements beyond titanium. Complications of this kind are observed especially among such elements as vanadium, chromium, manganese, columbium, molybdenum, tantalum, tungsten and uranium.

Another factor that complicates the chemistry of the elements of high atomic weight is the general tendency to form secondary valence compounds, especially by the elements of small atomic volume, such as those in the so-called eighth group. All the elements of high atomic weight such as antimony, bismuth, selenium, tellurium, iodine and cesium, show very marked tendencies to form secondary valence compounds. A general discussion of this field, however, would be out of place here, 


\section{Valence, Coördination Number and Covalence.}

According to the octet theory each carbon atom in a molecule of an organic substance has an octet and shares all 4 pairs of its electrons with adjacent atoms. For organic compounds, therefore, a pair of electrons held in common by two atoms corresponds exactly to the bond in the ordinary valence theory. Among other compounds, however, this relationship does not hold. Thus the octet theory indicates that the nitrogen atom in nitric acid shares 4 pairs of electrons with the oxygen atoms, in other words, the valence of nitrogen is 4 . To distinguish between the valence thus found and that assumed in the ordinary valence theory we shall denote by the term "covalence" the number of pairs of electrons which a given atom shares with its neighbors.

Werner's coördination number represents the number of atoms, or molecules, irrespective of their valency, which are arranged in space around a given atom. The maximum coördination number for carbon is 4 which is realized in saturated hydrocarbons and halogen compounds. In many organic substances; however, such as carbon dioxide, formaldehyde, etc., the coördination number is less than 4 . From the standpoint of the octet theory, the ordinary conception of valence is not definite, but involves at least 3 different properties of the atoms. In tracing general relationships between the elements it has usually been necessary to disregard all valences of the elements, except the maximum positive and negative valences. Now the maximum positive valence is a definite conception-it represents the number of electrons in the shell of the atom. Thus if the element combines with an excess of fluorine or oxygen these elements will usually take all the electrons in the shell. The maximum number of fuorine atoms or twice the maximum number of oxygen atoms thus held is a direct measure of the total number of available electrons in the shell. On the other hand, the maximum negative valence represents the number of electrons which the atom must take up to reach a stable form like that of the inert gases. Both of these conceptions are definite, although quite different. In most compounds, however, the atoms do not take up or give up electrons but rather share them with other atoms.

With carbon it so happens that the number of pairs of electrons shared by other atoms is equal both to the maximum positive and to the maximum negative valence. For other elements, however, there is no necessary relation between the number of pairs of electrons shared and the number of electrons in the shell of the original atom. It is for this reason that the utmost confusion occurs when the ordinary valences are applied to inorganic compounds in which atoms share pairs of electrons.

In using the octet theory to determine the structure of inorganic compounds we determine $p$ in Equation 2 from the total number of 
available electrons and make no assumptions regarding covalence. This simple theory corresponds with the known facts very much better than the ordinary valence theory, but does not yet accomplish all that could be desired in explaining why certain compounds exist, while others do not. For example, since phosphorus and nitrogen atoms contain the same number of electrons in their shells, the simple octet theory represented by Equation 2, indicates that nitrogen compounds corresponding to all known phosphorus compounds could exist and vice versa. Thus we might expect the following compounds: $\mathrm{H}_{3} \mathrm{NO}_{4}, \mathrm{Na}_{4} \mathrm{~N}_{2} \mathrm{O}_{7}$, $\mathrm{P}_{2} \mathrm{O}$. Similarly because sulfur and oxygen have equal numbers of electrons in their shells we might expect compounds like $\mathrm{OS}_{2}, \mathrm{H}_{2} \mathrm{OS}_{4}, \mathrm{OF}_{6}$, and $\mathrm{O}_{2} \mathrm{Cl}_{2}$ corresponding to $\mathrm{SO}_{2}, \mathrm{H}_{2} \mathrm{SO}_{4}, \mathrm{SF}_{6}$ and $\mathrm{S}_{2} \mathrm{Cl}_{2}$, respectively.

The octet theory may be made much more useful by supplementing it by a study of the values of the covalence as observed for the different elements. We have already noticed that with carbon it is practically always 4 while with fluorine it is usually one or zero. Table VII gives in the column marked $P$ a list of the values of covalence corresponding to the elements of the two short periods. The symbol $(\mathrm{o}+)$ means that the atom does not share any pairs of electrons with other atoms but has given up one or more electrons and therefore has become positively charged as, for example, in the case of the lithium ion. The symbol (o-) indicates that the atom has taken up electrons to complete its shell but does not share electrons with other atoms, as, for example, the chlorine ion $\mathrm{Cl}^{-}$.

TABLE VII.

Covalence of the First 18 Elements.

\begin{tabular}{|c|c|c|c|c|c|c|c|c|c|c|c|c|c|c|c|c|}
\hline Ele- & $\begin{array}{c}E . \\
\text { Elec- } \\
\text { trons } \\
\text { in } \\
\text { in }\end{array}$ & & & $\underset{\text { ovalen }}{P}$ & & & & Ele- & $\begin{array}{c}E . \\
\text { Elec- } \\
\text { trons } \\
\text { in } \\
\text { shell. }\end{array}$ & & & oval & & & & \\
\hline $\mathrm{H}$ & I & $(0+)$ & I ( & - & & . & . & & & & & & & & & \\
\hline $\mathrm{He}$ & 0 & o & .. & $\ldots$ & & . & . & $\mathrm{Ne}$ & 0 & 0 & . & . & . & . & . & \\
\hline $\mathrm{Li}$ & I & $(o+)$ & .. & . . & & . & . & $\mathrm{Na}$ & I & $(0+)$ & . & . & . & & $\cdots$ & \\
\hline $\mathrm{Be}$ & 2 & $(0+)$ & $4 ?$ & $\ldots$ & & . & . & $\mathrm{Mg}$ & 2 & $(0+)$ & . & . & - & & . & \\
\hline B & 3 & $(0+)$ & 4 & $\ldots$ & • & . & . & $\mathrm{Al}$ & 3 & $(o+)$ & 4 & . & • & . & . & \\
\hline C & 4 & . & 4 & . & - & . & 3 & $\mathrm{Si}$ & 4 & $(0+)$ & 4 & . & • & . & $\ldots$ & \\
\hline $\mathbf{N}$ & 5 & . & 4 & 3 & 2 & . & 3 & $\mathrm{P}$ & 5 & $(0+)$ & 4 & 3 & . & . & $\ldots$ & \\
\hline O & 6 & . & . & 3 & 2 & I & . 2 & $\mathrm{~S}$ & 6 & $(0+)$ & 4 & 3 & 2 & I & $(0-)$ & \\
\hline $\mathrm{F}$ & 7 & .. & . & . & • & I (c & $\longrightarrow)_{I}$ & $\mathrm{Cl}$ & 7 & . & 4 & 3 & 2 & $\mathbf{I}$ & $(0-)$ & \\
\hline $\mathrm{Ne}$ & 0 & 0 & $\ldots$ & . & . & . & . . & Ar & 0 & $\mathbf{o}$ & . & . & . & . & $\ldots$ & \\
\hline
\end{tabular}

The values of $P$ represent the number of pairs of electrons which the atom shares with other atoms. In the column marked $S$ is given the maximum number of pairs of electrons which an atom is capable of sharing with a single other atom. The values of $P$ shown by heavy faced type represent the covalence which occurs in the largest numbers 
of compounds. Thus for nitrogen in ammonium salts, in nitrates, in $\mathrm{NO}_{4}$, etc., $P=4$, while in ammonia, nitrous acid, $\mathrm{NCl}_{3}$, and many organic compounds, etc., $P=3$.

This table brings out clearly how the covalence of the elements from carbon to fluorine decreases steadily as the number of electrons approaches that of neon. With the corresponding elements of the second short period the lower limits of the covalence decrease as in the first period, but the maximum covalence remains constant at 4 . Thus chlorine forms $\mathrm{HClO}_{4}$, but there is no corresponding fluorine compound.

The covalence of an atom is closely related to the coördination number. According to Werner the coördination number is 4 in the following compounds: $\mathrm{NH}_{4} \mathrm{Cl}, \mathrm{HBF}_{4}, \mathrm{H}_{2} \mathrm{SO}_{4}, \mathrm{H}_{3} \mathrm{PO}_{4}, \mathrm{HPH}_{2} \mathrm{O}_{2}, \mathrm{H}_{2} \mathrm{PHO}_{3}$, $\mathrm{HClO}_{4}$, but not in $\mathrm{HNO}_{3}, \mathrm{CO}_{2}$ or $\mathrm{CH}_{2} \mathrm{O}$. According to the octet theory the covalence of the central atom of all these compounds is 4 . In $\mathrm{HNO}_{3}, \mathrm{CO}_{2}$ and $\mathrm{CH}_{2} \mathrm{O}$ one or more of the oxygen atoms is held to the central atom by two pairs of electrons, while in all the others there is never more than one pair of electrons involved in holding together two adjacent atoms. This difference between the octet theory and Werner's theory accounts for many of the cases of unsaturated supplementary valences.

In a very great number of compounds the coördination number and the covalence are identical and the octet theory then corresponds exactly to Werner's theory just as for organic compounds it is equivalent to the ordinary valence theory.

A tew examples will make this clear. In the compound $\mathrm{HBF}_{4}$ we have $n=5, \varepsilon=32, p=4$. Each fluorine atom thus shares a pair of electrons with the octet of the boron atom. The covalence of boron is thus 4 in this compound. Similarly for $\mathrm{NH}_{4} \mathrm{Cl}$ we place $n=2, e=16, p=0$. The 4 hydrogen nuclei thus attach themselves to the 4 pairs of electrons in the nitrogen octet making the positive ion $\mathrm{NH}_{4}+$. Since the chlorine atom las a complete octet it exists as a negative ion. Therefore ammonium chloride is a salt resembling sodium chloride. In this case also the central atom nitrogen has a covalence of four. These structures correspond exactly to those given by Werner.

An interesting compound whose constitution is not given by the ordinary valence theory is $\mathrm{B}\left(\mathrm{CH}_{3}\right)_{3} \mathrm{NH}_{3}$. Applying the octet theory we have $n=5, e=32, p=4$; whence the structure is<smiles>CC(C)(C)N</smiles>

According to the octet theory this is a typical primary valence compound in no way different from organic compounds. It is interesting to note that in this compound the covalence of both the carbon and nitrogen atoms is 4. The structure arrived at is identical with that postulated 
by Werner except that he assumes that the bond between the boron and the nitrogen corresponds to supplementary valence, while the others are of the primary type. The octet theory indicates that they are all of the same type.

For the compound $\mathrm{B}\left(\mathrm{CH}_{3}\right)_{3}$ we place $n=4, e=24$ and find $p=4$. But it is not possible to hold 3 methyl groups by 4 pairs of electrons. However, if we place $n=3$ we find $p=0$. The structure of this compound therefore must be $\mathrm{B}^{+++}\left[\mathrm{CH}_{3}\right]_{3}{ }^{-}$. Since the volume of the boron atom is small compared with the methyl group and since there are enough methyl groups to surround the boron atom, the electric field will be nearly wholly internal and the substance thus has a low boiling point and is not an electrolyte.

The compound $\mathrm{P}\left(\mathrm{CH}_{3}\right)_{3}$ gives $n=4, e=26, p=3$, so that each methyl group shares a pair of electrons with the phosphorus atom. This compound can add itself to methyl iodide to give a compound $\mathrm{P}\left(\mathrm{CH}_{3}\right)_{4} \mathrm{I}$, for which we find $n=6, e=40, p=4$. This leads to the structure $\left[\mathrm{P}\left(\mathrm{CH}_{3}\right)_{4}\right]^{+} \mathrm{I}^{-}$in which each of the 4 carbon atoms shares one of the pairs of electrons with the phosphorus atom. The electro-valence of both the carbon and the phosphorus atoms is 4 . The structure is quite analogous to that of typical primary valence compounds.

The structures of compounds like $\mathrm{KAuCl}_{4}, \mathrm{KBiCl}_{4}, \mathrm{~K} 2 \mathrm{Zn}(\mathrm{CN})_{4}$, $\mathrm{Cu}\left(\mathrm{NH}_{3}\right)_{4} \mathrm{Cl}_{2}, \mathrm{~K}_{2} \mathrm{NiF}_{4}, \mathrm{Pt}\left(\mathrm{PCl}_{3}\right)_{2} \mathrm{Cl}_{2}$, etc., can be found by the octet theory in the same way. As a final example let us consider the platino-ammonia compounds. In these the platinum is divalent, that is, there are two available electrons in the shell of the atom. For the compound $\mathrm{Pt}\left(\mathrm{NH}_{3}\right)_{4} \mathrm{Cl}_{2}$ we place $n=7, e=48, p=4$. The $4 \mathrm{NH}_{3}$ radicals are thus held directly to the platinum each sharing a pair of electrons. This allows the nitrogen and the platinum to have a covalence of 4 . The chlorine atoms become ions. For the compound $\left[\mathrm{Pt}\left(\mathrm{NH}_{3}\right)_{3} \mathrm{Cl}\right] \mathrm{Cl}$ we place $n=6, e=40, p=4$. The $3 \mathrm{NH}_{3}$ radicals and one of the chlorine atoms are attached to the platinum while the second chlorine forms an ion. The compound $\mathrm{Pt}\left(\mathrm{NH}_{3}\right)_{2} \mathrm{Cl}_{2}$ gives $n=5, e=32, p=4$ so that both chlorine atoms are attached to the platinum. For $\mathrm{K}\left[\mathrm{PtNH}_{3} \mathrm{Cl}_{3}\right]$ we have $n=5, e=32, p=4$. All 3 chlorine atoms and the ammonia are attached to the platinum, and the potassium forms a positive ion. In $\mathrm{K}_{2} \mathrm{PtCl}_{4}$ we find $n=5, e=32, p=4$ so that all 4 chlorine atoms are held by the platinum while the potassium atoms form positive ion. It will be noted that in each of these compounds the covalence of the nitrogen and the platinum is 4. According to this theory all these compounds should be looked upon as typical primary valence compounds.

Compounds with Coördination Number 6.-The elements of the first short period never have a coördination number greater than 4 . This is probably due to the strong tendency to complete the first octet. Among 
the elements of the second period silicon forms $\mathrm{H}_{2} \mathrm{SiF}_{6}$ and a few other compounds which show a coördination number of 6 even when these compounds are in solution. Aluminum forms compounds like cryolite, $\mathrm{Na}_{3} \mathrm{AlF}_{6}$, but this exists only in the solid state. Phosphorus, sulfur and chlorine form no compounds of this kind with the exception of the compound $\mathrm{SF}_{6}$.

The elements from titanium to nickel and the corresponding elements in the subsequent periods, have especially strong tendencies to form compounds with a coördination number 6 . With these elements there is little or no tendency to complete an octet unless all 4 pairs of electrons are shared, so it is not surprising that a larger number of pairs of electrons can also be taken up. We may imagine two ways in which this may occur. It is possible that a shell of $x 2$ electrons consisting of 6 pairs tends to form around the central atom. We may call such a group a disextet. If $m$ is the number of disextets in a molecule then we have by analogy with Equation 2

$$
p=1 / 2(12 m+8 n-e) .
$$

We may use this equation for compounds in which one of the atoms has a coördination number 6 in the same way that Equation 2 was used for compounds with coördination number 4 .

The other way of looking at these compounds is to consider, as we did in the case of $\mathrm{H}_{2} \mathrm{SiF}_{6}$, that the central atom does not share any pairs of electrons with the surrounding atoms, but holds these by electrostatic forces. It is evident that there is no difficulty in explaining the structure of $\mathrm{K}_{2} \mathrm{PtCl}_{6}$ on the assumption that the platinum atom has 4 positive charges and that the 6 chlorine ions are held around it by electrostatic attraction. On the other hand, it is not at first apparent how groups like $\mathrm{NH}_{3}, \mathrm{H}_{2} \mathrm{O}$, etc., can be held by electrostatic forces in compounds like $\left[\mathrm{Pt}\left(\mathrm{NH}_{3}\right)_{6}\right] \mathrm{Cl}_{4}$ and $\mathrm{PtCl}_{4}\left(\mathrm{H}_{2} \mathrm{O}\right)_{2}$. The groups that can enter into compounds in this way are $\mathrm{NH}_{3}, \mathrm{H}_{2} \mathrm{O}, \mathrm{HCl}, \mathrm{PCl}_{3}$, etc., in which the covalences are less than 4 .

We may look upon each of these groups as consisting of a central octet to which are attached positive radicals. In the cases of $\mathrm{NH}_{3}$, $\mathrm{H}_{2} \mathrm{O}$ and $\mathrm{HCl}$ the hydrogen nucleus is the positive element. In $\mathrm{PCl}_{3}$ the central octet has 8 electrons having a kernel with only 5 positive charges, so that it has a net negative charge of 3 units. Each chlorine atom contributes to this structure 6 electrons and a kernel with 7 positive charges, a net positive charge equal to one.

If we now assume that the positive parts of these added substances are mobile, then when the molecule is brought near a positive charge the central atom is attracted by this while the others are repelled. Thus a molecule of water normally represented by $\mathrm{HOH}$, when it is brought near a positively charged ion will take the form 
$\mathrm{H}_{\mathrm{H}}^{\mathrm{O}}$.....Pt $\mathrm{Pt}^{++++}$. This displacement of the positive charges in the water molecule causes it to be strongly attracted to charged ions, particularly those having large charges.

Because of this effect any highly charged ion, especially if of small volume, can attract molecules of such substances as water, ammonia, etc. The number that can be held depends on geometrical considerations. In view of the more or less cubical form of most atoms and the symmetry with which 6 groups can place themselves it is not surprising that the coördination number of 6 should be so common.

By either of these theories we can account for the structure of practically all complex compounds having a coördination number 6 . The second of these theories explains also the few cases in which the coördination number has values other than 4 or 6 .

\section{General Conclusions.}

The theory of atomic structure advanced in the present paper not only explains in a satisfactory manner the general properties and relationships of all the elements, but also gives a theory of the formation and structure of compounds which agrees excellently with the facts. It leads directly to a valence theory for organic compounds which is the exact equivalent of the ordinary theory. When applied to the structure of complex inorganic compounds it leads to a theory practically identical with that of Werner. In cases like those of the oxides of nitrogen, etc., which have not previously been explained by any theory of valence the results are thoroughly satisfactory. The structure of the nitrogen, carbon monoxide and hydrocyanic acid molecules are accounted for and new relationships are obtained.

Under these conditions the postulates underlying the theory receive strong support. In fact, the results seem to establish the fundamental correctness of most of the postulates. The recent advances in the physics of the electron have been largely along the lines of Bohr's theory. It is generally assumed that the electrons are revolving all in one plane, in orbits about the nucleus. Such a view is wholly inconsistent with that of the present paper. Bohr's theory has had marked success in explaining and even in predicting new facts connected with the spectra of hydrogen, helium and lithium, and must therefore contain important elements of truth.

It will probably be possible to reconcile the two theories. As has already been pointed out, Bohr's stationary states have a close resemblance to the cells postulated in the present theory. The series of numbers I, $1 / 4,1 / 9,1 / 25$ occur in much the same way in both theories.

The cellular structure postulated here also seems to be closely related to 
J. J. Thomson's ${ }^{1}$ theory of atomic structure in which he postulates tubes of force. It seems as though each cell in the present theory is analogous to the inner end of one of Thomson's cylindrical tubes of force. This view suggests that in an atom the electrons are acted on by a repulsive force inversely proportional to the cube of the distance from the nucleus and an attractive force proportional to $\mathrm{I} / \tau^{2}$ where $\tau$ is the index number of the shell in which the electron is located. Thus instead of the force varying continuously, as in Coulomb's law, it varies discontinuously in proportion to $1,1 / 4,1 / 9,1 / 25$, etc., and only at large distances where $\tau$ is very large does the force vary approximately continuously. In some such way we may hope to be led to a modification of Bohr's theory in which the electrons do not rotate about the nucleus.

\section{Summary.}

The theory presented in this paper is essentially an extension of Lewis' 2 theory of the "cubical atom." It may be most concisely stated in terms of the following postulates:

1. The electrons in atoms are either stationary or rotate, revolve or oscillate about definite positions in the atom. In the most stable atoms, namely those of the inert gases, the electrons have positions symmetrical with respect to a plane, called the equatorial plane, passing through the nucleus at the center of the atom. No electrons lie in the equatorial plane. There is an axis of symmetry (polar axis) perpendicular to this plane through which 4 secondary planes of symmetry pass forming angles of $45^{\circ}$ with each other. These atoms thus have the symmetry of a tetragonal crystal.

2. The electrons in any given atom are distributed through a series of concentric (nearly) spherical shells, all of equal thickness. Thus the mean radii of the shells form an arithmetric series $1,2,3,4$, and the effective areas are in the ratios $1: 2^{2} ; 3^{2} ; 4^{2}$.

3. Each shell is divided into cellular spaces or cells occupying equal areas in their respective shells and distributed over the surface of the shells according to the symmetry required by Postulate $x$. The first shell thus contains 2 cells, the second 8 , the third I8, and the fourth 32 .

4. Each of the cells in the first shell can contain only one electron, but each other cell can contain either one or two. All the inner shells must have their full quotas of electrons before the outside shell can contain any. No cell in the outside layer can contain two electrons until all the other cells in this layer contain at least one.

5. Two electrons in the same cell do not repel nor attract one another with strong forces. This probaly means that there is a magnetic at-

1 Phil. Mag., 26, 792, x044 (I9I3).

a This Journai, 38,762 (I916). 
traction (Parson's magnetic theory) which nearly counteracts the electrostatic repulsion.

6. When the number of electrons in the outside layer is small the arrangement of the electrons is determined by the (magnetic?) attraction of the underlying electrons. But when the number of electrons increases, especially when the layer is nearly complete, the electrostatic repulsion of the underlying electrons and of those in the outside shell becomes predominant.

7. The properties of the atoms are determined primarily by the number and arrangement of electrons in the outside shell and by the ease with which the atom is able to revert to more stable forms by giving up or taking up electrons.

8. The stable and symmetrical arrangements of electrons corresponding to the inert gases are characterized by strong internal and weak external fields of force. The smaller the atomic number, the weaker the external field.

9. The most stable arrangement of electrons is that of the pair in the helium atom. A stable pair may also be held by: (a) a single hydrogen nucleus; (b) two hydrogen nuclei; $(c)$ a hydrogen nucleus and the kernel of another atom; (d) two atomic kernels (very rare).

Io. The next most stable arrangement of electrons is the octet, that is, a group of 8 electrons like that in the second shell of the neon atom. Any atom with atomic number less than 20 , and which has more than 3 electrons in its outside layer tends to take up enough electrons to complete its octet.

I I. Two octets may hold one, two, or sometimes 3 pairs of electrons in common. One octet may share one, two, 3 , or 4 pairs of its electrons with one, two, 3 , or 4 other octets. One or more pairs of electrons in an octet may be shared by the corresponding number of hydrogen nuclei. No electron can be shared by more than two octets.

This theory explains the periodic properties of all the elements including those of the eighth group and the rare earths. It meets with success in explaining the magnetic properties of the elements, and applies as well to the so-called physical properties, such as boiling points, freezing points, electric conductivity, etc., as it does to the "chemical properties." It leads to a simple theory of chemical valence for both polar and non-polar substances. In the case of organic compounds the results are identical with those of the ordinary valence theory, while with oxygen, nitrogen, chlorine, sulfur, and phosphorus compounds, the new theory applies as well as to organic compounds, although the ordinary valence theory fails nearly completely.

This theory explains also the structure of compounds which, according to Werner's theory, are second order compounds with a coördination 
number equal to 4. According to the present theory, such compounds are to be regarded rather as typical primary valence compounds.

This valence theory is based on the following simple equation:

$$
c=8 n-2 p
$$

where $e$ is the total number of available electrons in the shells of all the atoms in a molecule; $w$ is the number of octets forming the outside shells, and $p$ is the number of pairs af electrons held in common by the octets. This equation is a complete mathematical statement of the primary valence requirements, not only in organic, but in inorganic chemistry.

The theory leads to very definite conceptions as to the positions of the electrons in the molecules or space lattices of compounds. The structures of molecules of nitrogen, carbon monoxide, hydrogen cyanide, and NO prove to be exceptional in that the kernels of both atoms in the molecule are contained within a single octet. This accounts for the practically identical "physical" properties of nitrogen and carbon monoxide, and for the abnormal inertness of molecular nitrogen.

The results obtained by the use of the postulates are so striking that one may safely reason that the results establish the fundamental correctness of the postulates.

These conclusions, however, are not easily reconciled with Bohr's theory of the atom. Bohr's stationary states have a rather close resemblance to the cellular structure postulated in the present theory. There are also striking points of similarity with J. J. Thomson's theory of the structure of atoms, in which he assumes that the attractive forces are limited to certain tubes of ioree.

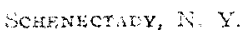

[CONTRIBUTION FROM THE CHEMISTRY DEPARTMENT, UNIVERSITY OF EDINBURGH.]

\section{MANGANOUS TARTRATE AND POTASSIUM MANGANOUS TARTRATE. \\ BY LEONARD DOBBIN. \\ Received February 25, 1919.}

\section{Manganous Tartrate.}

Manganous tartrate was first prepared by Scheele, ${ }^{1}$ who obtained it by the interaction of a solution of potassium tartrate with a solution of a manganous salt, but did not describe it. Bergman ${ }^{2}$ also mentions this interaction, while J. B. Richter ${ }^{3}$ employed it, using hot solutions, as his method of freeing manganese from iron--a purpose for which it was like-

" Kongl. Vetenskapsacad. Handingar, 35, 95 (1774).

" "Phys. and Chem. Fisays" (Trans. by Cullen), 2, 22 I (1784).

${ }^{3}$ Nenere Gegenstände der Chemie, Erstes Stïck, I79r, 13. 32, and Crell's Chem. Ann., 1796, Bd. II, 13. 300. 